\title{
Bayesian inference of earthquake rupture models using polynomial chaos expansion
}

\author{
Hugo Cruz-Jiménez ${ }^{1}$, Guotu $\mathrm{Li}^{2}$, Paul Martin Mai ${ }^{1}$, Ibrahim Hoteit ${ }^{1}$, and Omar M. Knio ${ }^{1,2}$ \\ ${ }^{1}$ King Abdullah University of Science and Technology, Thuwal 23955, Saudi Arabia \\ ${ }^{2}$ Department of Mechanical Engineering and Materials Science, Duke University, Durham, NC 27708, USA
}

Correspondence: Guotu Li (guotu.li@duke.edu) and Omar M. Knio (omar.knio@kaust.edu.sa)

Received: 8 January 2018 - Discussion started: 5 February 2018

Revised: 13 June 2018 - Accepted: 30 June 2018 - Published: 31 July 2018

\begin{abstract}
In this paper, we employed polynomial chaos (PC) expansions to understand earthquake rupture model responses to random fault plane properties. A sensitivity analysis based on our PC surrogate model suggests that the hypocenter location plays a dominant role in peak ground velocity (PGV) responses, while elliptical patch properties only show secondary impact. In addition, the PC surrogate model is utilized for Bayesian inference of the most likely underlying fault plane configuration in light of a set of PGV observations from a ground-motion prediction equation (GMPE). A restricted sampling approach is also developed to incorporate additional physical constraints on the fault plane configuration and to increase the sampling efficiency.
\end{abstract}

\section{Introduction}

One of the most important challenges seismologists and earthquake engineers face in designing large civil structures (e.g., buildings, dams, bridges, power plants) and response plans, especially in highly populated cities prone to large damaging earthquakes, is the reliable estimation of groundmotion characteristics at a given location. Ground-motion prediction equations (GMPEs), which are one of the most important elements for probabilistic seismic hazard analysis (PSHA), are designed for this purpose. These are obtained from regression analysis by fitting a dataset (empirical and simulated) and are mainly expressed in terms of site conditions, source-site distance (e.g., rupture distance or JoynerBoore distance, denoted as $R_{\mathrm{JB}}$ distance hereafter ${ }^{1}$ ), magni-

\footnotetext{
${ }^{1}$ The Joyner-Boore distance is defined as the shortest distance from a site to the surface projection of the rupture plane.
}

tude, and mechanism, although other terms such as directivity and hanging-wall effect are also considered (Abrahamson et al., 2014). The equations can be derived for peak ground displacement (PGD), peak ground velocity (PGV), peak ground acceleration (PGA), and spectral acceleration (SA) for a damping of $5 \%$ at different periods. Ideally, an optimal GMPE has to be robust and include physical terms to avoid overfitting the data, which can result in the inclusion of too many parameters. When other effects are considered (such as amplitude and duration of rupture directivity; Somerville et al., 1997) or more data are available (Atkinson and Boore, 2011), GMPEs are modified to better explain attenuation patterns.

Many efforts have been made to characterize the seismic ground-motion considering both real and simulated data. For example, using real data, five research groups under the Pacific Earthquake Engineering Research Center Next Generation Attenuation (PEER NGA) project derived GMPEs for shallow crustal earthquakes considering an extensive database of recorded ground motion (Chiou et al., 2008). Later, Arroyo and Ordaz (2010a, b) obtained GMPEs using both synthetic data and two subsets of accelerograms of the NGA database (Chiou et al., 2008). Arroyo and Ordaz (2010b) highlighted the necessity to merge finite fault modeling (Atkinson and Silva, 2000) with observations to obtain GMPEs that better predict the amplitudes in zones where data are insufficient. Verification and validation studies (Maufroy et al., 2015, 2016) were also conducted in a large effort to understand ground motion and showed the importance of both accurate source parameters and the geological description of the medium to reproduce observed ground motion. Singh et al. (2017) improved the agreement between 
observed ground motion and GMPEs by including site effects of the area. Numerical simulations have also helped to explain ground-motion characteristics. For instance, Furumura and Singh (2002) described attenuation patterns for both deep in-slab and shallow interplate earthquakes, while Cruz-Jiménez et al. (2009) explained ground-motion amplification due to a volcanic layer. Mahani and Atkinson (2012) modeled the decay of spectral amplitudes in several locations in North America.

In this study, we investigate the level of complexity needed in kinematic rupture models of magnitude 6.5 strike-slip events to produce ground motion similar to a reference GMPE. To this end, we utilize the polynomial chaos (PC) approach (Ghanem and Spanos, 1991; Xiu and Karniadakis, 2002; Le Maître and Knio, 2010) to build functional representations of PGV responses of an original source model. Thanks to the significant reduction in computational cost of the PC surrogate models (in comparison with both the original source model and a Bayesian analysis based on Markov chain Monte Carlo (MCMC) sampling, which requires a prohibitive number of model runs; Minson et al., 2014), it is suitable to utilize the PC surrogates in a Bayesian inference framework (Sudret and Mai, 2013; Sraj et al., 2016; Giraldi et al., 2017). This enables us to quantitatively rank different kinematic source models given by the PGVs they produce and identify the most likely one that fits a chosen reference GMPE (expectation). The ranking considers uncertainties in both the GMPE and model parameters. This provides useful insight on the level of complexity needed in kinematic source models for ground-motion simulations to satisfy both observational constraints and engineering/design requirements for seismic safety.

This paper is organized as follows. In Sect. 2, we provide detailed descriptions of the source model configurations, including the calculation of synthetic seismograms. In Sect. 3, we present the PC analysis of PGVs as a function of random variations of the kinematic models, including the validation of PC surrogate models and discussions of various statistical quantities. In Sect. 4, we conduct a PC-based Bayesian inference analysis to identify the most likely kinematic rupture model that best fits a chosen GMPE reference curve. Finally, we conclude our key findings and propose potential improvements for future work in Sect. 5.

\section{Source model}

A magnitude $M_{\mathrm{w}}=6.5$ strike-slip earthquake (seismic moment $6.31 \times 10^{18} \mathrm{Nm}$; rake $=0^{\circ}$ ) on a single-segment vertical fault plane is considered. The fault plane is chosen to be a rectangle with fixed length $L=27 \mathrm{~km}$ and width $W=10 \mathrm{~km}$, which are the most frequent values among 100 sample realizations following scaling relations (e.g., Wells and Coppersmith, 1994; Mai and Beroza, 2000; Thingbaijam et al., 2017). The top of the fault plane is located $2 \mathrm{~km}$ below

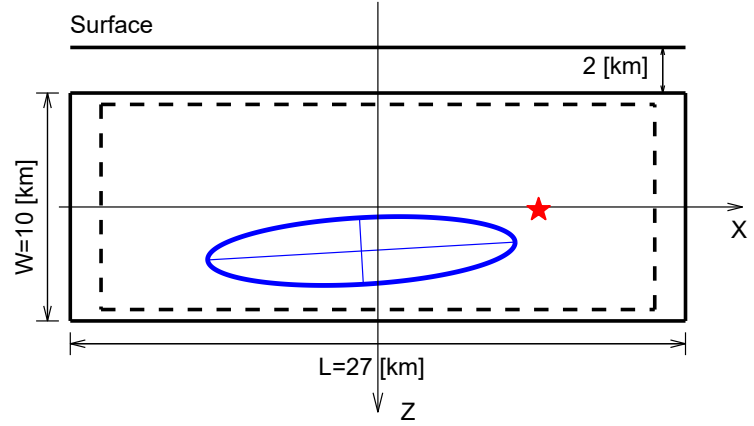

Figure 1. Example of fault plane configuration. The red star denotes hypocenter location, and the ellipse is the asperity with Gaussian slip distribution inside. The slip distribution is tapered in the area between the dashed and solid rectangles.

the ground surface. Figure 1 shows an example configuration of the fault plane, in which the red star denotes the hypocenter and the ellipse is the asperity with Gaussian slip distribution inside. The maximum slip $S_{\max }$ is chosen such that the mean slip (over the entire fault plane) remains constant $(0.71 \mathrm{~m})$ when varying the ellipse size (which ensures that the moment magnitude remains constant, $M_{\mathrm{w}}=6.5$ ). The slip between the elliptical patch boundary and dashed rectangle (Fig. 1) is set to be $S_{\max } / e$ (where $e$ is the Euler number), the minimum value at the patch boundary from the Gaussian slip distribution. The slip between the solid and dashed rectangles (the horizontal and vertical gaps are $5 \%$ of the length and width of the fault plane, respectively) is tapered to avoid non-physical slip patterns. The entire fault plane is discretized in along-strike and down-dip directions with a grid size of $0.02 \mathrm{~km}$. We use a regularized Yoffe function (Tinti et al., 2005) with a rise time $\operatorname{Tr}=1.25 \mathrm{~s}$ following sourcescaling relations (Somerville et al., 1999) and slip acceleration time $t_{\text {acc }}=0.225 \mathrm{~s}$, as suggested by Tinti et al. (2005). At each node of the discretized fault plane, we assign $\mathrm{Tr}, t_{\mathrm{acc}}$, slip-rate in along-strike and down-dip directions, and rupture time. We consider a rupture speed of $0.75 V_{\mathrm{s}}$ (where the shear wave speed $V_{\mathrm{s}}$ is listed in Table 1) in all source models.

PGVs at a virtual network of $N_{\text {obs }}=56$ stations (Fig. 2) are calculated from synthetic seismograms of the two horizontal components of ground motion at each site for a large set of source rupture models. We use COMPSYN (Spudich and $\mathrm{Xu}, 2003$ ), a code based on the discrete wavenumber/finite element method proposed by Olson et al. (1984) to calculate the synthetic seismograms up to a maximum frequency of $1.5 \mathrm{~Hz}$ at each station of the virtual array. COMPSYN solves the equation of motion considering initial conditions of zero displacement and velocity at a reference time $t_{0}$ and specifying traction or displacement on the bounding surface of the medium (boundary conditions) using the unit outward normal vector (details about the scheme can be seen in Olson et al., 1984). The grid resolution used in COMPSYN is variable and uses a spacing of one-sixth of the mini- 


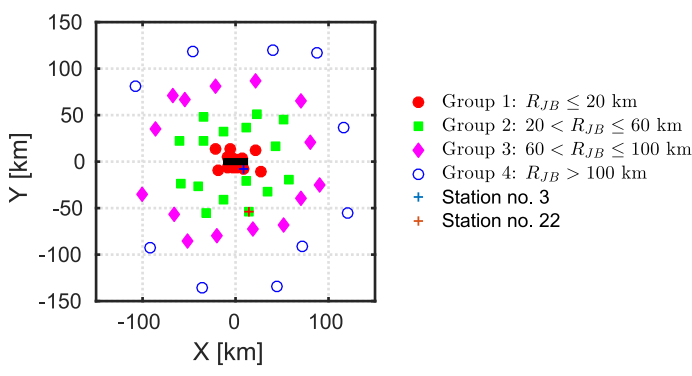

Figure 2. A virtual network of $N_{\text {obs }}=56$ stations where PGV responses are reported by the source model. The solid black line at the center denotes the length and location of the fault plane. Note that the 56 stations are grouped into four sets (indicated by different colors/symbols) according to their $R_{\mathrm{JB}}$ distances (see details in Sect. 4).

Table 1. Velocity model used in this study, modified from Boore et al. (1997).

\begin{tabular}{rrr}
\hline Depth $(\mathrm{km})$ & $V_{\mathrm{p}}\left(\mathrm{km} \mathrm{s}^{-1}\right)$ & $V_{\mathrm{s}}\left(\mathrm{km} \mathrm{s}^{-1}\right)$ \\
\hline 0 & 2.4 & 1.5 \\
0.5 & 4.4 & 2 \\
1.5 & 5.3 & 2.7 \\
2.5 & 5.5 & 2.9 \\
4 & 5.7 & 3.3 \\
8 & 6.1 & 3.5 \\
14 & 6.8 & 3.9 \\
16.6 & 7.1 & 4.1 \\
27 & 8 & 4.6 \\
350 & 8.2 & 4.65 \\
\hline
\end{tabular}

mum shear wavelength at depth $z$. The grid extends to a total depth that depends on the wavenumber, which means that the maximum depth decreases when the wavenumber increases. This approach considers a layered 1-D velocity structure. We apply the velocity model shown in Table 1, which corresponds to a slightly modified version of the generic model by Boore et al. (1997) for California. The resulting PGVs serve as our quantities of interest (QoIs, each denoted as $\mathcal{Q}_{j}$, for $j=1,2, \ldots, N_{\text {obs }}$ ). We aim at understanding stochastic source model PGV responses to random fault plane configurations of the source process (slip distributions and hypocenter location). To this end, we consider variations in seven physical parameters listed in Table 2, which parameterize the fault plane configurations, i.e., locations of both the hypocenter and elliptical asperity patch, as well as its shape and orientation. We restrict the hypocenter and elliptical patch to be inside the fault plane and limit the area ratio (AR) of the elliptical patch to the entire fault plane $(L \times W)$ between 5 and $29 \%$. These restrictions lead to nonlinear dependency between feasible ranges of different physical parameters (see Appendix A for more details).
Table 2. Parameters governing fault plane configurations.

\begin{tabular}{|c|c|c|}
\hline Index & Parameter & Physical interpretation \\
\hline 1 & $\mathrm{AR}$ & $\begin{array}{l}\text { Area ratio, } \mathrm{AR}=\frac{\pi a b}{L \times W} \in \\
{[0.05,0.29]}\end{array}$ \\
\hline 2 & $x_{\mathrm{h}}(\mathrm{km})$ & $\begin{array}{l}x \text { coordinate of the hypocenter } x_{\mathrm{h}} \in \\
{[-13.5,13.5]}\end{array}$ \\
\hline 3 & $z_{\mathrm{h}}(\mathrm{km})$ & $\begin{array}{l}z \text { coordinate of the hypocenter } z_{\mathrm{h}} \in \\
{[-5,5]}\end{array}$ \\
\hline 4 & $a(*)(\mathrm{km})$ & $\begin{array}{l}\text { Semi-major axis } a \in \\
{\left[\sqrt{\frac{\mathrm{AR} \cdot L \cdot W}{\pi}}, L / 2\right]}\end{array}$ \\
\hline 5 & $\theta(*)$ & $\begin{array}{l}\text { Inclination angle of the elliptical } \\
\text { patch }\end{array}$ \\
\hline 6 & $x_{\mathrm{c}}\left({ }^{*}\right)(\mathrm{km})$ & $\begin{array}{l}x \text { coordinate of the center of } \\
\text { elliptical patch }\end{array}$ \\
\hline 7 & $z_{\mathrm{c}}\left({ }^{*}\right)(\mathrm{km})$ & $\begin{array}{l}z \text { coordinate of the center of } \\
\text { elliptical patch }\end{array}$ \\
\hline
\end{tabular}

* denotes parameters whose feasible ranges are dependent on others.

\section{Polynomial chaos framework}

PC expansions (Ghanem and Spanos, 1991; Xiu and Karniadakis, 2002; Le Maître and Knio, 2010) ${ }^{2}$ are used in this study to understand earthquake rupture model responses (in terms of PGVs) to random configurations of slip distribution and hypocenter location. We associate each of the physical parameters with a canonical PC random variable $\xi_{i}$ $\left(i \in\left\{1,2, \ldots, n_{\mathrm{d}}\right\}\right.$, where $n_{\mathrm{d}}=7$ is the stochastic space dimension) and assume all $\xi_{i}$ values are independent and uniformly distributed over $[-1,1]$. That is, the joint distribution of the random parameter vector $\xi$ is

$p(\xi)= \begin{cases}2^{-7} & \text { if } \boldsymbol{\xi} \in \mathbf{\Xi} \equiv[-1,1]^{7}, \\ 0 & \text { otherwise. }\end{cases}$

Each random parameter vector $\xi \in \boldsymbol{\Xi}$ can be linked uniquely to a realization of the physical parameter vector (see mapping details in Appendix A). We thus focus on constructing functional representations of PGV responses at each station with respect to the canonical variable $\xi$, which parameterize the physical parameters in Table 2 . It is worth mentioning that the mapping from canonical random variable $\xi$ to physical fault plane configuration parameters does not lead to uniform distributions for physical parameters, due to their interdependency as indicated in Table 2. Nevertheless, the validity of PC expansions based on canonical random variable $\xi$ is well maintained, as suggested by the cross-validation and empirical error analyses later in this section.

Let $\mathcal{Q}_{j}(\xi)$ be the PGV response to $\xi$ at the $j$ th station $\left(j \in\left\{1,2, \ldots, N_{\text {obs }}\right\}\right)$, and assume each $\mathcal{Q}_{j}$ is a second-order

\footnotetext{
${ }^{2} \mathrm{An}$ open-source toolkit for the PC framework is available at http://www.sandia.gov/UQToolkit/ (last access: 20 December 2017) (Debusschere et al., 2004, 2016).
} 
random variable, i.e., $\mathcal{Q}_{j}(\boldsymbol{\xi})$ is in the Hilbert space $L_{2}(\boldsymbol{\Xi}, p)$, and

$\mathbb{E}\left[\mathcal{Q}_{j}^{2}\right]=\int_{\Xi} \mathcal{Q}_{j}(\xi)^{2} p(\boldsymbol{\xi}) \mathrm{d} \boldsymbol{\xi}<+\infty$,

$\forall j \in\left\{1,2, \ldots, N_{\text {obs }}\right\}$.

One can approximate $\mathcal{Q}_{j}(\xi)$ using a truncated PC expansion as follows:

$\mathcal{Q}_{j}(\xi) \approx \widetilde{\mathcal{Q}}_{j}(\xi)=\sum_{\alpha=0}^{N_{\mathrm{p}}} c_{\alpha} \boldsymbol{\Psi}_{\alpha}(\boldsymbol{\xi}), \quad \forall j \in\left\{1,2, \ldots, N_{\mathrm{obs}}\right\}$,

where $N_{\mathrm{p}}$ is a truncation parameter and $\left(N_{\mathrm{p}}+1\right)$ is the number of expansion terms retained in the PC surrogate models. In this study, we truncated the PC expansion at total polynomial order of $q=9$. Given $n_{\mathrm{d}}=7$, one can calculate the total number of polynomials via

$N_{\mathrm{p}}+1=\frac{\left(q+n_{\mathrm{d}}\right) !}{q ! n_{\mathrm{d}} !}=11440$.

By adopting the classical convention of $\boldsymbol{\Psi}_{0}(\boldsymbol{\xi})=1$, the mean and variance of a PC surrogate $\mathcal{Q}_{j}(\xi)$ can be expressed as

$\mathbb{E}[\widetilde{\mathcal{Q}}]=\sum_{\alpha=0}^{N_{\mathrm{p}}} c_{\alpha}\left\langle\boldsymbol{\Psi}_{\alpha}, 1\right\rangle=c_{0}$,

and

$$
\begin{aligned}
\mathbb{V}[\widetilde{\mathcal{Q}}] & =\mathbb{E}\left[(\widetilde{\mathcal{Q}}-\mathbb{E}[\widetilde{\mathcal{Q}}])^{2}\right] \\
& =\sum_{\alpha, \beta=1}^{N_{\mathrm{p}}} c_{\alpha} c_{\beta}\left\langle\boldsymbol{\Psi}_{\alpha}, \boldsymbol{\Psi}_{\beta}\right\rangle=\sum_{\alpha=1}^{N_{\mathrm{p}}} c_{\alpha}^{2}\left\|\boldsymbol{\Psi}_{\alpha}\right\|_{L_{2}}^{2},
\end{aligned}
$$

where $\langle\cdot\rangle$ denotes the inner product in the Hilbert space $L_{2}(\boldsymbol{\Xi}, p)$ with respect to the joint distribution $p(\boldsymbol{\xi})$ (Le Maître and Knio, 2010).

To determine the expansion coefficients ( $c_{\alpha}$ values) in Eq. (3), we rely on a Latin hypercube sample (LHS) (McKay et al., 1979) set (denoted as $\mathcal{P}_{\text {LHS }}$ hereafter) of $N_{\text {LHS }}=8000$ earthquake rupture model realizations through $\left\{\boldsymbol{\xi}_{k}\right\}_{1 \leq k \leq N_{\mathrm{LHS}}}$, and solve the following basis pursuit denoising (BPDN) problem (Van Den Berg and Friedlander, 2007, 2008) ${ }^{3}$ at each station:

$\boldsymbol{c}^{*}=\arg \min _{\boldsymbol{c} \in \mathbb{R}^{N_{\mathrm{p}}+1}}\|\boldsymbol{c}\|_{l_{1}}$ s.t. $\left\|\mathcal{Q}_{j}-[\boldsymbol{\Psi}] \boldsymbol{c}\right\| \leq \gamma\left\|\mathcal{Q}_{j}\right\|_{l_{2}}$,

$\forall j \in\left\{1,2, \ldots, N_{\text {obs }}\right\}$,

where $\mathcal{Q}_{j}=\left(\mathcal{Q}_{j}\left(\boldsymbol{\xi}_{1}\right), \mathcal{Q}_{j}\left(\boldsymbol{\xi}_{2}\right), \ldots, \mathcal{Q}_{j}\left(\boldsymbol{\xi}_{N_{\mathrm{LHS}}}\right)\right)^{T}$ is the model PGV realization vector at the $j$ th station, and $c \in \mathbb{R}^{N_{\mathrm{p}}+1}$ is the coefficient vector for the corresponding PC

\footnotetext{
${ }^{3}$ The corresponding source code is available at https://github. com/mpf/spgl1 (last access: 20 December 2017).
}

surrogate model. $[\Psi] \in \mathbb{R}^{N_{\mathrm{LHS}} \times\left(N_{\mathrm{p}}+1\right)}$ denotes the polynomial matrix with each element $[\Psi]_{i, \alpha}=\Psi_{\alpha}\left(\boldsymbol{\xi}_{i}\right)$. Note that $[\Psi]$ is station invariant. The scalar parameter $\gamma$ indicates the model noise level and is determined numerically via a $k$-fold $(k=5)$ cross-validation process (Seber and Lee, 2012) over a discrete grid of $\gamma=\left\{10^{-4}, 10^{-3}, 10^{-2}: 0.005: 0.2\right\}$.

Following Sobol (1993), Homma and Saltelli (1996), variance-based first-order and total-order sensitivity indices associated with a subset of random variables $(i \subset$ $\left.\left\{1,2, \ldots, n_{\mathrm{d}}\right\}\right)$ can be calculated, respectively, as follows:

$\mathbb{S}_{i}=\frac{\sum_{\alpha \in \mathcal{S}_{i}} c_{\alpha}^{2}\left\|\boldsymbol{\Psi}_{\alpha}\right\|_{L_{2}}^{2}}{\sum_{\alpha=1}^{N_{\mathrm{p}}} c_{\alpha}^{2}\left\|\boldsymbol{\Psi}_{\alpha}\right\|_{L_{2}}^{2}}$

$\mathbb{T}_{i}=\frac{\sum_{\alpha \in \mathcal{T}_{i}} c_{\alpha}^{2}\left\|\boldsymbol{\Psi}_{\alpha}\right\|_{L_{2}}^{2}}{\sum_{\alpha=1}^{N_{\mathrm{p}}} c_{\alpha}^{2}\left\|\boldsymbol{\Psi}_{\alpha}\right\|_{L_{2}}^{2}}$,

where $\mathbb{S}_{i}$ (first-order sensitivity) is the relative variance contribution of those polynomials (denoted as index set $\mathcal{S}_{i}$ ) exclusively related to random variables in the subset $\boldsymbol{i}$, while $\mathbb{T}_{\boldsymbol{i}}$ (total-order sensitivity) is the relative variance contribution of polynomials (denoted as index set $\mathcal{T}_{\boldsymbol{i}}$ ) involving any of the random variables in $\boldsymbol{i}$ (including cross polynomials between variables in $\boldsymbol{i}$ and its complement $\left.\boldsymbol{i}_{\sim}, \boldsymbol{i} \cup \boldsymbol{i}_{\sim}=\left\{1,2, \ldots, n_{\mathrm{d}}\right\}\right)$. Note that by definition the two polynomial index sets satisfy $\mathcal{S}_{i} \subset \mathcal{T}_{i}$

\subsection{Validation of PC models}

We first validate our PC surrogate models for PGVs at all stations. To this end, we introduce a second independent source model simulation ensemble (again an 8000-member LHS set $\mathcal{P}_{\text {LHS }}^{\text {valid }} \subset \boldsymbol{\Xi}$ ) for the purpose of validation. (Note that $\mathcal{P}_{\text {LHS }}^{\text {valid }}$ is independent of the training set $\mathcal{P}_{\text {LHS }}$.) The following relative $l_{2}$ error is then examined for PGVs at each station.

$\epsilon_{j}=\sqrt{\frac{\sum_{k=1}^{N_{\mathrm{LHS}}}\left(\widetilde{\mathcal{Q}}_{j}\left(\boldsymbol{\xi}_{k}\right)-\mathcal{Q}_{j}\left(\boldsymbol{\xi}_{k}\right)\right)^{2}}{\sum_{k=1}^{N_{\mathrm{LHS}}} \mathcal{Q}_{j}\left(\boldsymbol{\xi}_{k}\right)^{2}}}$,

$\forall j \in\left\{1,2, \ldots, N_{\text {obs }}\right\}$,

where $\widetilde{\mathcal{Q}}_{j}\left(\boldsymbol{\xi}_{k}\right)$ and $\mathcal{Q}_{j}\left(\boldsymbol{\xi}_{k}\right)$ denote PC and source model responses, respectively, to $\xi_{k}$ at the $j$ th station. $\xi_{k} \in \mathcal{P}_{\text {LHS }}$ or

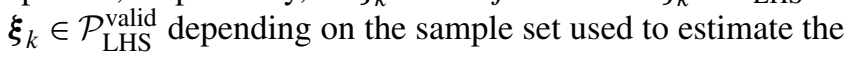
errors.

Figure 3 shows error estimates of PC surrogate models over the training set ( $\mathcal{P}_{\text {LHS }}$, blue dots) and the validation set $\left(\mathcal{P}_{\text {LHS }}^{\text {valid }}\right.$, red dots). It is not surprising to see slightly larger error estimates on the validation set, as the PC reconstruction process is unaware of this dataset. However, because almost all error estimates fall below $10 \%$ range, and in light of the close agreement (about $4 \%$ difference) between the blue and red dots, our PC surrogate models are deemed to suitably reproduce source model PGV responses throughout the entire station network. 


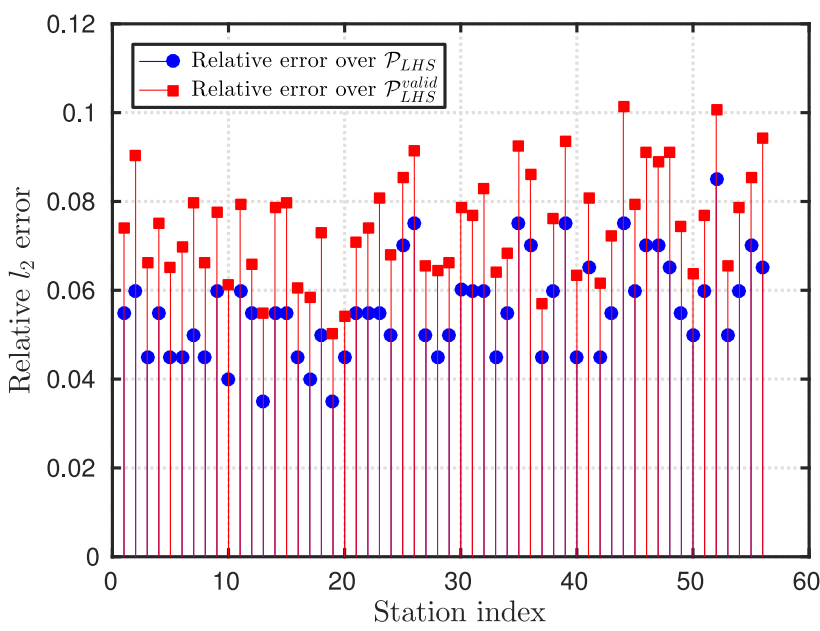

Figure 3. Relative $l_{2}$ errors of PC surrogate models. The crossvalidation errors are close to the error estimated from validation set. For brevity, we omit the cross-validation errors in the plot.

Apart from the above error estimates, the convergence of PC surrogate models with respect to truncation order is also investigated from a statistical point of view. Figure 4 shows PGV distributions from PC resampling on a 1 million member LHS set $\left(\mathcal{P}_{\text {LHS }}^{1 \mathrm{E} 6}\right)$ at two selected stations, with increasing odd PC truncation orders up to degree 9 . It is seen that when the truncation order is larger than 5, the difference in the PGV prediction distributions becomes relatively small, suggesting that our ninth-order PC expansions are sufficiently accurate for the source model under consideration.

We finally compare distributions of PC and source model predictions; see Fig. 5. It is observed that our PC surrogate models are capable of reproducing PGV distributions produced from source model realizations of the validation set $\mathcal{P}_{\text {LHS }}^{\text {valid }}$. Besides, the excellent agreement between the two PCpredicted distribution curves in Fig. 5 suggests that our existing 8000-model-simulation ensemble is statistically representative, which provides additional confidence in our PC representations.

\subsection{PC statistics}

The PC surrogate models obtained in the previous section provide immediate access to prediction statistics, as given by Eqs. (5) and (6). Figure 6 shows means and standard deviations of PC PGV predictions at different stations, along with a reference median PGV curve predicted by the GMPE in Boore and Atkinson $(2008)^{4}$. It is noted that two stations with

\footnotetext{
${ }^{4}$ The interested reader is referred to Mai (2009), http://www.opensha.org/glossary-attenuationRelation-BOORE_ ATKIN_2008 (last access: 20 December 2017) and http://www.gmpe.org.uk/gmpereport2014.pdf (last access: 20 December 2017) for more details on the GMPE employed in this paper.
}
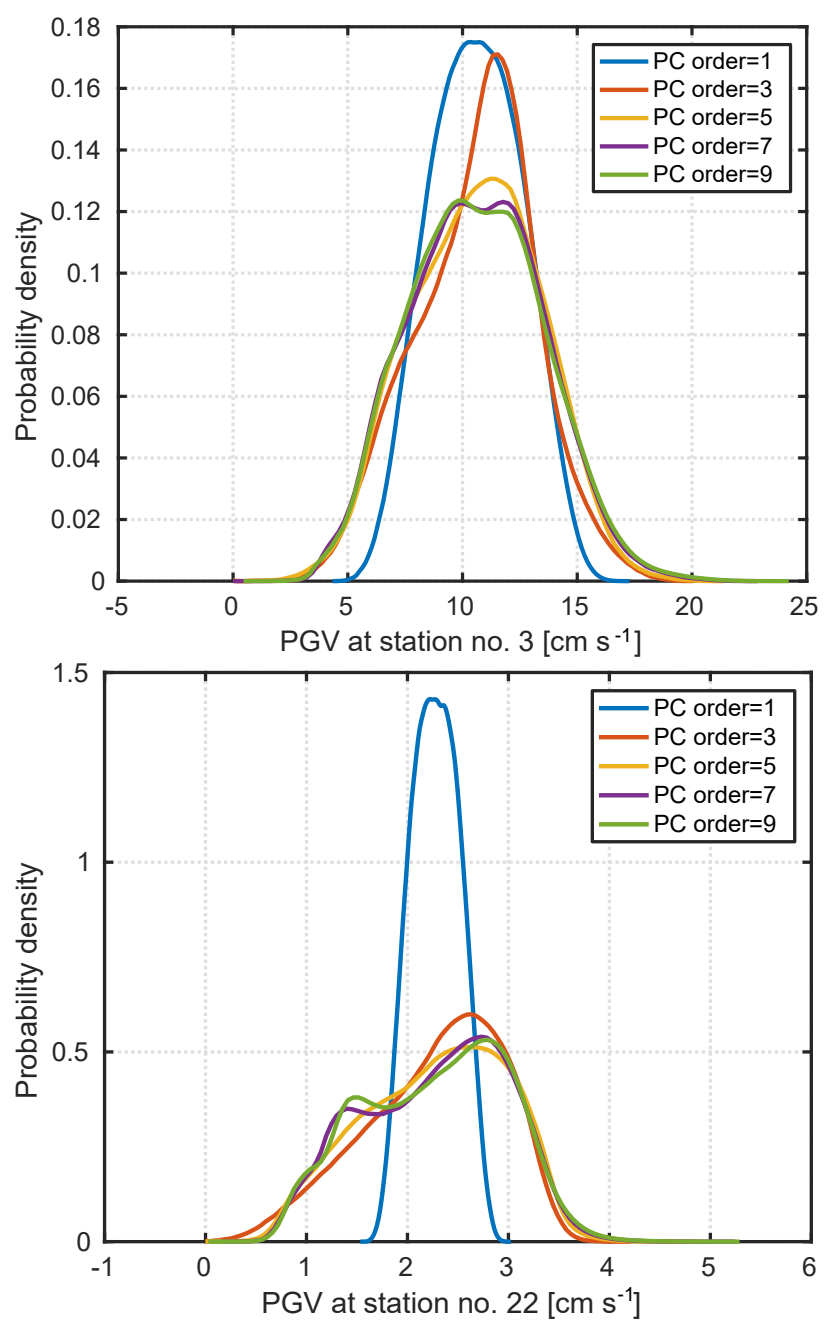

Figure 4. PC-predicted PGV distributions at two selected stations (as indicated in Fig. 2). Distribution curves are obtained using kernel density estimation (Sheather and Jones, 1991) from PC realizations on a 1 million member LHS set $\mathcal{P}_{\text {LHS }}^{1 \mathrm{E} 6}$.

similar $R_{\mathrm{JB}}$ distance can have very different PGV values. This is likely due to radiation-pattern effects, in particular directivity, which is addressed in great detail by Vyas et al. (2016). Besides, it is observed that PC predictions generally scatter around the GMPE curve. Though one should not expect an exact match between PC statistic and GMPE predictions, due to the difference in random sources underlying the two approaches, and the uninformative uniform canonical PC parameter distributions used to generate PC statistics, it is worth noting that the similar range of PC and GMPE predictions enables us to use the GMPE results as "observations" for the purpose of parameter inference discussed in Sect. 4. One can also observe that PGVs are generally largest near the fault plane and decrease with increasing $R_{\mathrm{JB}}$ distance. The overall tendency of PC prediction uncertainty (quanti- 

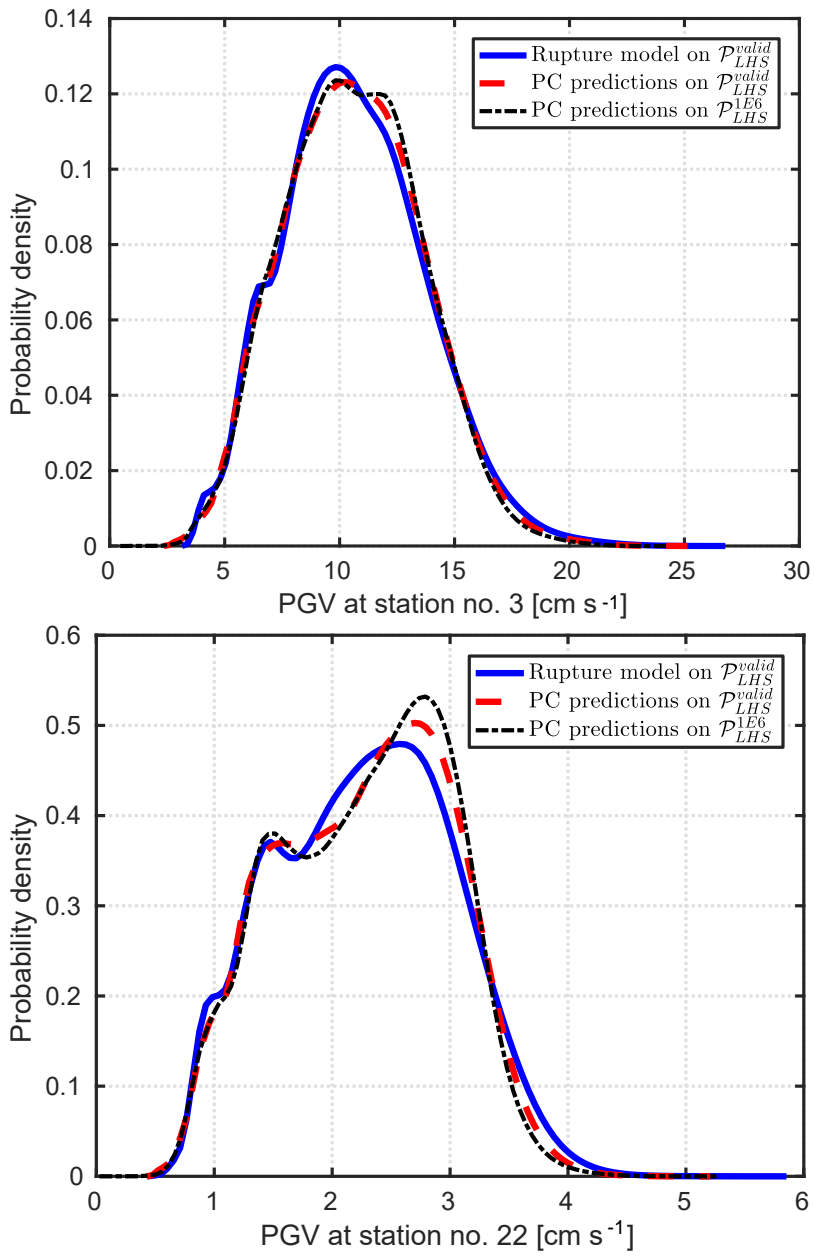

Figure 5. Comparison of PGV distributions predicted by the source model (blue solid curve) and PC surrogate model (red dashed curve), respectively, at selected stations (as indicated in Fig. 2) over the validation sample set $\mathcal{P}_{\text {LHS }}^{\text {valid }}$. The black dash-dotted curves are PGV prediction distributions obtained from PC surrogate model realizations on a 1 million member LHS set $\mathcal{P}_{\text {LHS }}^{1 \text { E6 }}$. Distributions are obtained using kernel density estimations (Sheather and Jones, 1991).

fied by the standard deviation bars) seems to decrease with increasing $R_{\mathrm{JB}}$ distance as well.

The conditional mapping from canonical PC random variables $(\xi)$ to physical fault plane configurations makes it difficult to isolate the relative impact of individual parameters. To address this difficulty, we rely on the global sensitivity analysis (Homma and Saltelli, 1996; Sobol, 1993) and discuss the statistical significance of each canonical random parameters in the rupture model.

Figure 7 shows both the first- and total-order sensitivity indices associated with each random parameter at different stations. These sensitivity indices reveal that the model PGV response is most sensitive to the location of the hypocenter ( $x_{\mathrm{h}}$ is dominant and $z_{\mathrm{h}}$ plays a secondary role) throughout

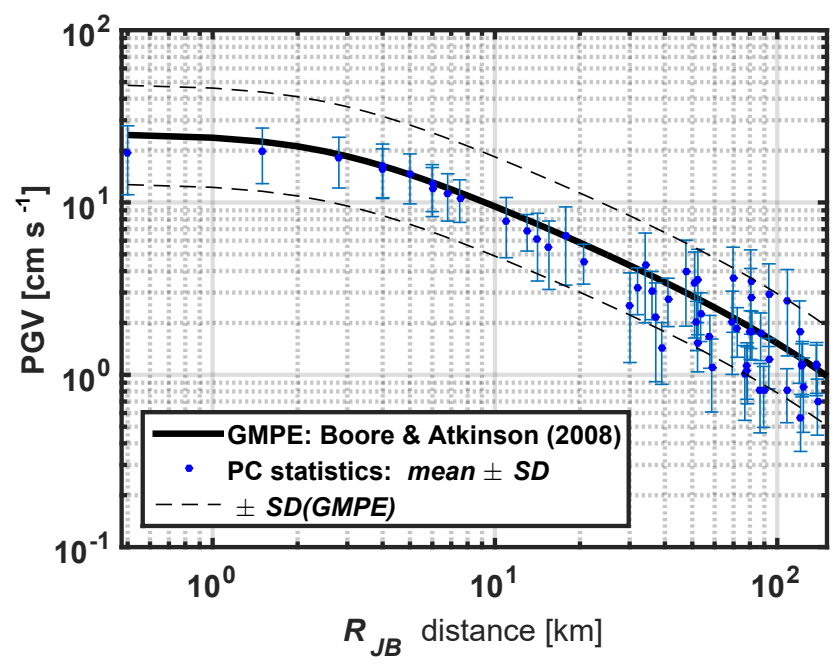

Figure 6. Comparison of PC statistics (based on uniform distribution assumption of the canonical PC random parameters) with GMPE results. Solid black curve denotes the median GMPE prediction, while the dashed lines are GMPE standard deviation bounds. Note that log scales are used in the plot.
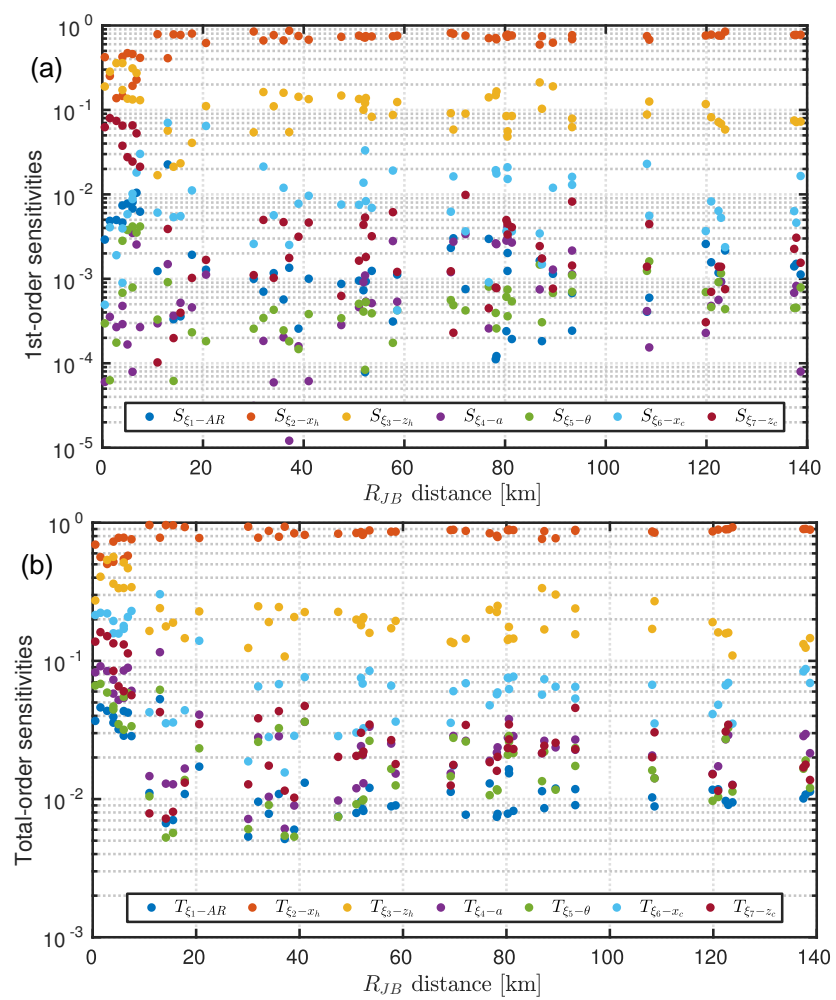

Figure 7. First- (a) and total-order (b) sensitivity indices at each station.

all stations, whereas the remaining random parameters (associated with elliptical asperity patch) are relatively insignificant. While it might be reasonable to neglect the elliptical patch parameters' impact on PGV response variability at far 


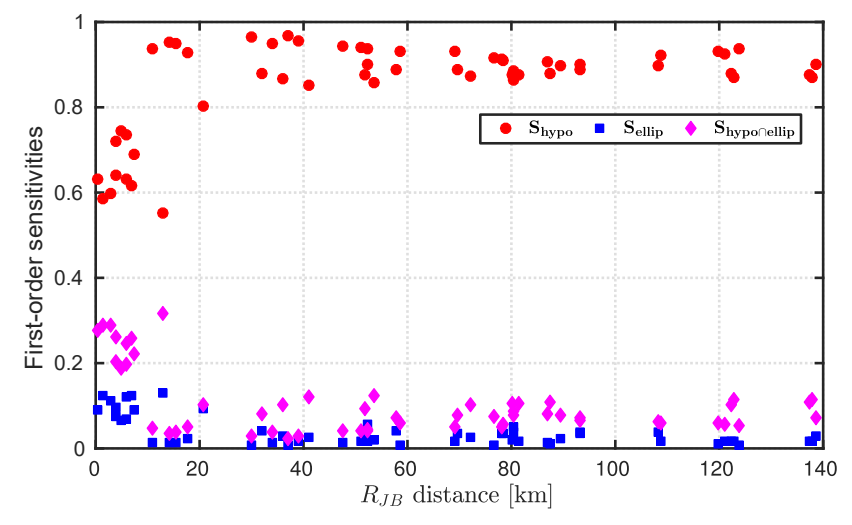

Figure 8. First-order sensitivity indices with respect to grouped parameters.

stations (with $R_{\mathrm{JB}}$ distance roughly more than $10 \mathrm{~km}$ away from the center), it is evident that at near-the-center stations, those elliptical patch parameters can still lead to a considerable impact on PGV response.

To better illustrate the above sensitivity observation, we divided the parameters into the following two groups $\left(\xi_{\text {hypo }}=\right.$ $\left\{\xi_{2}^{x_{\mathrm{h}}}, \xi_{3}^{z_{\mathrm{h}}}\right\}$ and $\xi_{\text {ellip }}=\left\{\xi_{1}^{\mathrm{AR}}, \xi_{4}^{\mathrm{a}}, \xi_{5}^{\theta}, \xi_{6}^{x_{\mathrm{c}}}, \xi_{7}^{z_{\mathrm{c}}}\right\}$; the superscripts denote the corresponding physical parameters) and calculated the first-order sensitivity indices associated with $\boldsymbol{\xi}_{\text {hypo }}$ and $\xi_{\text {ellip }}$ using Eq. (8a), denoted as $\mathbb{S}_{\text {hypo }}$ and $\mathbb{S}_{\text {ellip }}$, respectively. Note that the combined effect (interaction) of hypocenter location and elliptical patch parameters is simply given by $\mathbb{S}_{\text {hypoxellip }}=1-\mathbb{S}_{\text {hypo }}-\mathbb{S}_{\text {ellip }}$. The resulting group sensitivity indices are shown in Fig. 8. It is now clear that the hypocenter location alone is responsible for $80-90 \%$ of the variability in PGVs at distant stations. Meanwhile, near the center, the hypocenter location alone is associated with only $55-75 \%$ of the PGV variability, suggesting that the elliptical patch parameters play important roles with about 25-45\% contribution to the total PGV variability.

\section{Bayesian inference}

In this section, we utilize a Bayesian approach (Bernardo and Smith, 2001; Berger, 2013; Gelman et al., 2014) to find the most likely fault plane configuration, in the sense that the resulting earthquake rupture model produces PGVs that best match the reference GMPE curve for the same magnitude and focal mechanism (Boore and Atkinson, 2008). To this end, we first obtain the GMPE-predicted PGVs at the stations shown in Fig. 2, denoted as $\boldsymbol{d}$, which serve as observational data in our Bayesian inference, and compare $\boldsymbol{d}$ with our PC surrogate model predictions $\widetilde{\boldsymbol{d}}(\boldsymbol{\xi})=$ $\left(\widetilde{\mathcal{Q}}_{1}(\xi), \widetilde{\mathcal{Q}}_{2}(\xi), \ldots, \widetilde{\mathcal{Q}}_{N_{\text {obs }}}(\xi)\right)^{T}$.

\subsection{Bayesian formulation}

To formulate the Bayesian problem, we start with Bayes' formula:

$p(\boldsymbol{\eta} \mid \boldsymbol{d})=\frac{p(\boldsymbol{d} \mid \boldsymbol{\eta}) p(\boldsymbol{\eta})}{p(\boldsymbol{d})} \propto p(\boldsymbol{d} \mid \boldsymbol{\eta}) p(\boldsymbol{\eta})$,

where $\eta$ is the parameter vector to be inferred, $p(\eta)$ is the prior probability distribution of $\boldsymbol{\eta}$, and $p(\boldsymbol{d} \mid \boldsymbol{\eta})$ is the likelihood of observing $\boldsymbol{d}$ given $\boldsymbol{\eta}$. The denominator $p(\boldsymbol{d})$ is the marginal distribution known as evidence. (Note that this evidence can be neglected, as the MCMC sampling method (Haario et al., 2001; Roberts and Rosenthal, 2009) utilized below solely relies on the proportionality.) We adopt the assumption of independent Gaussian error at each station location; i.e., the discrepancy between observations (GMPEpredicted PGVs) and PC predictions at each station is an independent Gaussian variable:

$p\left(\epsilon_{j}\right)=p\left(d_{j}-\widetilde{d}_{j}\right)=\frac{1}{\sqrt{2 \pi \sigma^{2}}} \exp \left[-\frac{\left(d_{j}-\widetilde{d}_{j}\right)^{2}}{2 \sigma^{2}}\right]$,

$\forall j \in\left\{1,2, \ldots, N_{\text {obs }}\right\}$.

Recall that the PC prediction variability seems to decrease with $R_{\mathrm{JB}}$ distance according to Fig. 6. To account for this decay of PGV variance with $R_{\mathrm{JB}}$ distance in the Bayesian inference analysis, we partition the $N_{\text {obs }}$ stations into four groups according to their corresponding $R_{\mathrm{JB}}$ distances as indicated in Fig. 2, and associate each group of stations with a hyperparameter $\sigma_{l(j)}^{2}(l(j) \in\{1,2,3,4\}$ depending on the $R_{\mathrm{JB}}$ distance of the $j$ th station). As a result, the likelihood can be expressed as

$p(\boldsymbol{d} \mid \boldsymbol{\eta})=\prod_{j=1}^{N_{\mathrm{obs}}} \frac{1}{\sqrt{2 \pi \sigma_{l(j)}^{2}}} \exp \left(-\frac{\left(d_{j}-\widetilde{d}_{j}(\xi)\right)^{2}}{2 \sigma_{l(j)}^{2}}\right)$,

and accordingly the inference parameter vector $\boldsymbol{\eta}$ reads

$\boldsymbol{\eta}=\left(\xi_{1}, \xi_{2}, \ldots, \xi_{7}, \sigma_{1}^{2}, \sigma_{2}^{2}, \ldots, \sigma_{4}^{2}\right)^{T}$.

Our numerical experiments suggest that the $4-\sigma^{2}$ model above outperforms the model with only one hyperparameter for all stations. It is noted that we limit the number of uncertainty hyperparameters ( $\sigma_{i}^{2}$ values) to four in this study, due to the limited number of observations (PGVs at limited number of stations). If more observations were available, it might be beneficial to increase the number of hyperparameters.

The prior distribution of $\boldsymbol{\eta}$, without additional information on the model parameters, is usually given by assumptions of uniform distribution for canonical PC parameters $\xi$, and Jeffrey's priors (Sivia and Skilling, 2006) for hyperparameters $\sigma_{l}^{2}$ (as $\sigma_{l}^{2}$ is always greater than zero); consequently,

$p(\boldsymbol{\eta})= \begin{cases}\left(\frac{1}{2}\right)^{7} \prod_{l=1}^{4} \frac{1}{\sigma_{l}^{2}} & \forall \boldsymbol{\xi} \in \mathbf{\Xi} \text { and } \forall \sigma_{l}^{2}>0, \\ 0 & \text { otherwise, }\end{cases}$ 
and Bayes' rule reduces to

$$
\begin{aligned}
& p(\boldsymbol{\eta} \mid \boldsymbol{d}) \propto p(\boldsymbol{d} \mid \boldsymbol{\eta}) p(\boldsymbol{\eta})= \\
& \left\{\begin{array}{cl}
\prod_{j=1}^{N_{\mathrm{obs}}} \frac{1}{\sqrt{2 \pi \sigma_{l(j)}^{2}}} \exp \left(-\frac{\left(d_{j}-\widetilde{d}_{j}(\boldsymbol{\xi})\right)^{2}}{2 \sigma_{l(j)}^{2}}\right) & \forall \xi \in \boldsymbol{\Xi} \text { and } \\
{\left[\left(\frac{1}{2}\right)^{7} \prod_{l=1}^{4} \frac{1}{\sigma_{l}^{2}}\right]} & \forall \sigma_{l}^{2}>0, \\
0 & \text { otherwise. }
\end{array}\right.
\end{aligned}
$$

We rely on the adaptive metropolis MCMC approach (Haario et al., 2001; Roberts and Rosenthal, 2009) to sample the above posterior distribution. It is worth noting that MCMC methods, despite the improved efficiency against traditional MC approaches, generally require a large number of samples (typically tens of thousands, and even larger depending on the dimensionality of the problem). This is one of the main reasons why we utilize $\mathrm{PC}$ techniques, as the use of the corresponding PC surrogates in the MCMC simulation leads to significant reduction in computational cost. In this study, the MCMC sample size for inference is set to $10^{6}$.

\subsection{Inference results}

As mentioned above, we exploit the PC surrogate models in Bayesian inference analysis and update the posterior distribution of random parameters $(\boldsymbol{\xi} \in \mathbf{\Xi})$, as well as PGV prediction uncertainties ( $\sigma_{l}^{2}$ values), in light of the GMPE observations. Figure 9 shows the posterior probability distributions of hyperparameters $\sigma_{l}^{2}(l \in\{1,2,3,4\})$. It is evident that $\sigma_{l}^{2}$ decreases with $R_{\mathrm{JB}}$ distance (from $l=1$ to $l=4$ ), which supports our previous ansatz from Fig. 6.

Similarly, we examine the sampling chains of PC random parameters $\xi_{i}(i \in\{1,2, \ldots, 7\})$. While some parameters (e.g., $\xi_{1}, \xi_{2}, \xi_{3}$, and $\xi_{6}$ ) yield very informative posterior distributions (not shown here), others look relatively less informative. It is noted that our goal is to estimate the posterior distributions of the physical parameters in Table 2, instead of the PC parameters. Thus, it is desired to map the $\xi$ chain into the corresponding physical configuration chain, before inferring the most likely fault plane configuration.

Figure 10 shows the posterior distributions of the physical parameters after mapping from the PC parameter chain of $\xi$, as well as the corresponding inference of the fault plane configuration (bottom right panel). It is observed that in light of the GMPE PGV observations (1) the hypocenter location $\left(x_{\mathrm{h}}\right.$ and $\left.z_{\mathrm{h}}\right)$ is well identified; (2) the size of the elliptical patch seems to be more likely near the lower bound of the prior; (3) the inclination angle of the elliptical patch, as well as the location of the patch, are less conclusive. For example, despite the clear peak in the inclination angle plot, the posterior distribution is relatively flat, suggesting limited information gain compared with the prior knowledge. Furthermore, the $x_{\mathrm{c}}$ distribution only shows the fact that the ellipse tends to be in the left half of the fault plane; the definite lo- cation of the elliptical patch (either $x_{\mathrm{c}}$ or $z_{\mathrm{c}}$ ) is ambiguous. These findings are generally consistent with the results of the sensitivity analysis. Since the model is primarily sensitive to the hypocenter location, perturbing the hypocenter location leads to more effective adjustment in PGV responses. On the other hand, elliptical patch parameters have relatively small impact on PGV variance, which calls for more observational data to pin down those parameters.

One needs to be cautious about the Bayesian inference results discussed above. From the physical point of view, the spatial distribution of those stations (see Fig. 2) where PGVs are reported is almost "symmetric" about the center of the fault plane ( $x=0$ and $y=0)$; as a result, one would expect to see a "symmetric" twin configuration that is roughly equally plausible from the Bayesian inference. However, this "symmetric" counterpart is clearly missing in the above inference results. This is probably because when MCMC chain converges to the high probability region of hypocenter location in the bottom right quadrant of the fault plane, it becomes more and more difficult to escape from this high probability region and explore the other side of parameter space. In other words, there could be bimodal structures in the distributions of $x_{\mathrm{h}}$ (as well as $x_{\mathrm{c}}$ ) which the previous MCMC process fails to identify (e.g., the configuration in which the hypocenter is located in the bottom left quadrant of the fault plane, and the ellipse is centered at somewhere on the right half of the fault plane). While in theory it is possible to identify the missing multimodal distributions of random parameters by further increasing the number of MCMC samples, the computational cost can be excessive. Alternatively, we verify our expectation of seeing the "symmetric" counterpart configuration by rerunning the MCMC simulation starting with the "symmetric" counterpart configuration (i.e., with hypocenter being in the bottom left quadrant of the fault plane, and elliptical patch being on the right side of the fault plane). The resulting fault plane configuration inference is shown in Fig. 11. As expected, the new MCMC process ended up with a fault plane configuration that is roughly "symmetric" to the previous inference result, especially for the hypocenter location. The asymmetric behavior of the elliptical patch stems from the fact that (1) the $N_{\text {obs }}$ stations are not exactly symmetrically distributed; thus, one should not expect exact symmetry; (2) as discussed before, the PGV responses are less sensitive to the elliptical patch properties, leading to ambiguity in inferring these properties.

\subsection{Inference with restricted prior}

The previous inference results are all based on almost complete ignorance of dependency between hypocenter location and the slip area (asperity). However, previous studies (Mai et al., 2005; Irikura and Miyake, 2011) suggested some constraints on the relative hypocenter location (Mai et al., 2005) with respect to the asperity, and size of the asperity (Irikura and Miyake, 2011). 


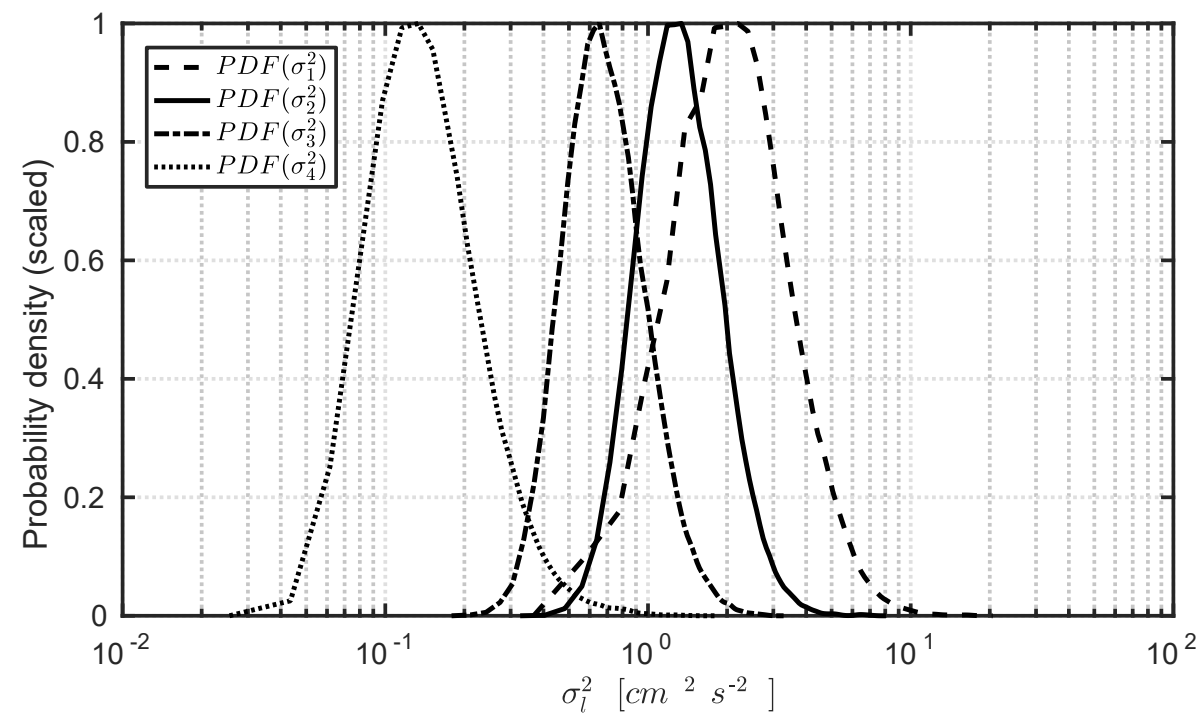

Figure 9. Posterior probability distributions of prediction uncertainty parameters (each PDF curve is scaled to have unit peak height for better comparison).

In this section, we consider the following restrictions in our inference analysis:

R-1. The elliptical patch is inside the dashed rectangle $\left(\left[L^{\prime}, W^{\prime}\right]=0.9 \times[L, W]\right)$ shown in Fig. 1.

R-2. The area ratio of the elliptical patch (AR) is between 15 and $29 \%$ of the fault plane area, i.e., $0.15<\mathrm{AR}<0.29$.

R-3. The elliptical patch is not too elongated, i.e., the axis ratio $\frac{a}{b} \leq 3$.

R-4. The hypocenter is located outside but near the elliptical patch, i.e., $x_{\mathrm{h}}=\left(a+3 \zeta_{\mathrm{h}_{1}}\right) \cos \left(2 \pi \zeta_{\mathrm{h}_{2}}\right)$ and $z_{\mathrm{h}}=$ $\left(b+b \frac{3}{a} \zeta_{\mathrm{h}_{1}}\right) \sin \left(2 \pi \zeta_{\mathrm{h}_{2}}\right) \forall\left(\zeta_{\mathrm{h}_{1}}, \zeta_{\mathrm{h}_{2}}\right) \in[0,1]^{2}$.

One of the advantages of having previous PC surrogate models (which were built based on uninformative prior that spans a wide range of feasible scenarios, i.e., minimal restrictions as in Table 2) is that the above four additional parameter restrictions can be efficiently performed a posteriori, namely without the need for performing new model simulations (Alexanderian et al., 2012).

To begin with, we first incorporate the above restrictions into the Bayesian framework, namely by modifying the previous prior distribution (Eq. 14) as follows:

$p^{*}(\boldsymbol{\eta})= \begin{cases}\left(\frac{1}{2}\right)^{7} \prod_{l=1}^{4} \frac{1}{\sigma_{l}^{2}} & \forall \boldsymbol{\xi} \in \mathbf{\Xi}, \forall \sigma_{l}^{2}>0 \\ & \begin{array}{l}\text { and all restrictions } \\ \text { are satisfied, } \\ \text { otherwise }\end{array}\end{cases}$

However, due to the strong restrictions listed above, the support of the above prior probability distribution (Eq. 16) turns out to be extremely limited in the parameter space $\Xi$, leading to computationally inefficient MCMC sampling (since most of the samples drawn from a proposal distribution will end up not satisfying at least one of the restrictions and thus zero prior probability). To mitigate the difficulty of inefficient sampling due to restricted prior distribution, we introduce a new layer of parameterization, mapping from $\Xi$ to restricted physical configurations. (Details on this new mapping mechanism are given in Appendix B.)

Figure 12 shows the MCMC process of drawing random samples from proposal distributions and calculates the resulting posterior probability. Without additional restrictions (orange path), the parameter vector $\zeta=\xi$, and the whole process reduces to the standard MCMC process we used in the previous section. By introducing the new parameterization process (see Algorithm 2), we are transforming the original problem, which is based on PC parameter vector $\xi$, into a new inference problem based on $\zeta$ (we denote $\zeta$ as the auxiliary random parameter vector hereafter, to distinguish it from the PC parameter vector $\xi$ ). This transformation is based on the mapping from $\zeta$ to $\xi$ (i.e., $\boldsymbol{\xi}=\boldsymbol{\xi}(\zeta)$ ) via their commonly associated physical configuration. For clarity, we formulate the new $\zeta$ based Bayesian problem as follows:

$$
\begin{aligned}
& p\left(\boldsymbol{\eta}^{*} \mid \boldsymbol{d}\right) \propto \\
& \begin{cases}{\left[\left(\frac{1}{2}\right)^{7} \prod_{l=1}^{4} \frac{1}{\sigma_{l}^{2}}\right] \prod_{j=1}^{N_{\mathrm{obs}} \frac{1}{\sqrt{2 \pi \sigma_{l(j)}^{2}}}}} & \\
\exp \left(-\frac{\left(d_{j}-\widetilde{d}_{j}(\xi(\zeta))\right)^{2}}{2 \sigma_{l(j)}^{2}}\right) & \forall \zeta \in \boldsymbol{\Xi}, \forall \sigma_{l}^{2}>0, \\
0 & \text { otherwise. }\end{cases}
\end{aligned}
$$

where $\eta^{*}=\left(\zeta_{1}, \zeta_{2}, \ldots, \zeta_{7}, \sigma_{1}^{2}, \sigma_{2}^{2}, \ldots, \sigma_{4}^{2}\right)^{T}$. 

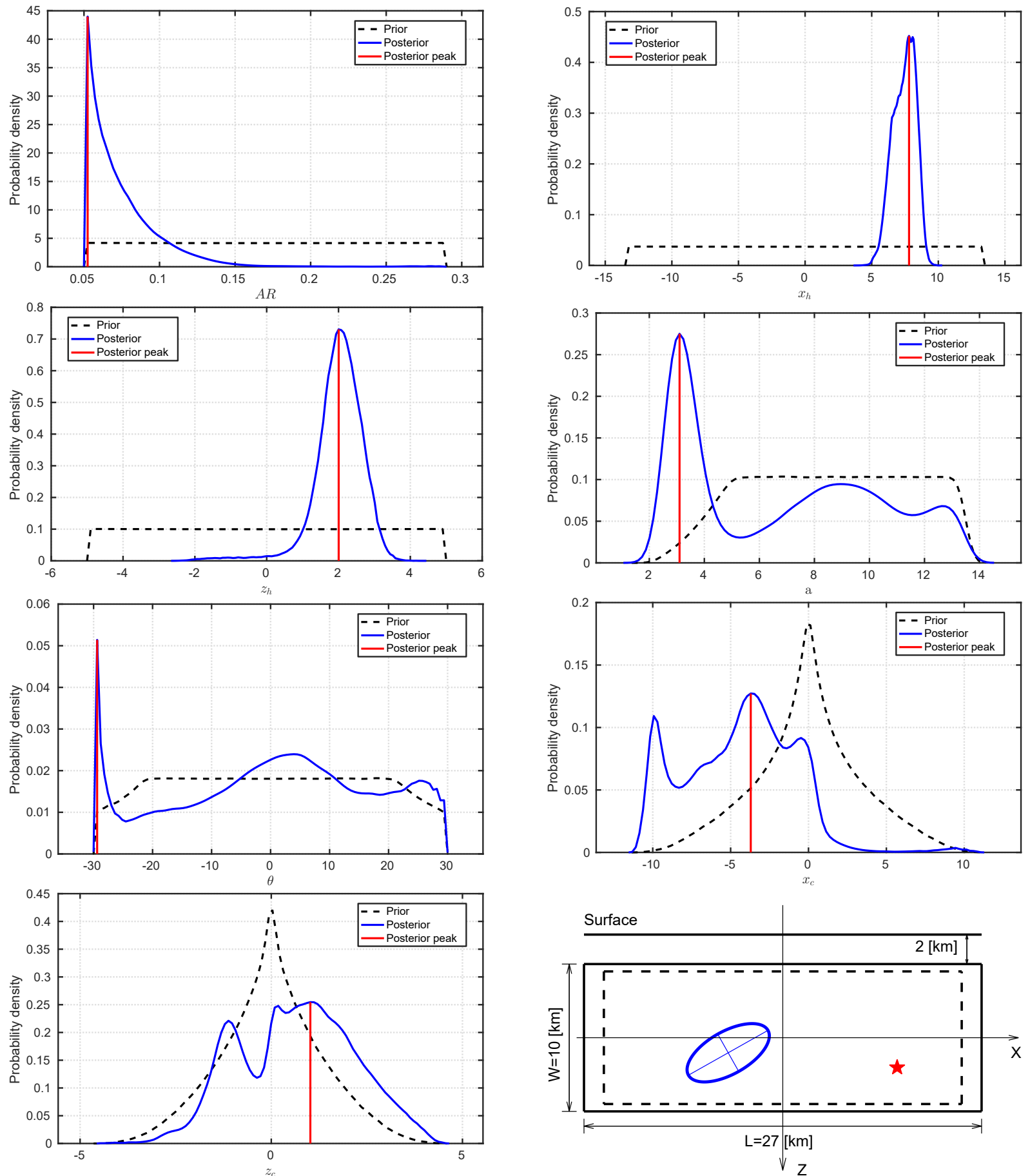

Figure 10. Prior (dashed black, derived from uniform $\boldsymbol{\xi}$ distribution in $\boldsymbol{\Xi}$ ) and posterior (solid blue) distributions of physical fault plane configuration parameters. The bottom right panel shows the inferred fault plane configuration.

Following the same analysis as discussed before, we show the inference results under restrictions in Fig. 13. Note that the prior distributions of those physical parameters are different from those in Fig. 10, as the new ones are derived from uniformly distributed auxiliary random vector $\zeta \in \mathbf{\Xi}$, instead of PC parameters $\boldsymbol{\xi} \in \boldsymbol{\Xi}$. Nevertheless, we see very consis- tent results of hypocenter location, as well as the location of the elliptical patch, compared with those in Fig. 10. The area ratio AR, though larger than the previous inferred value, still favors the lower end of the prescribed parameter range. The elliptical patch ends up with a larger area and longer semimajor axis (compared to the results in Figs. 10 and 11). These 


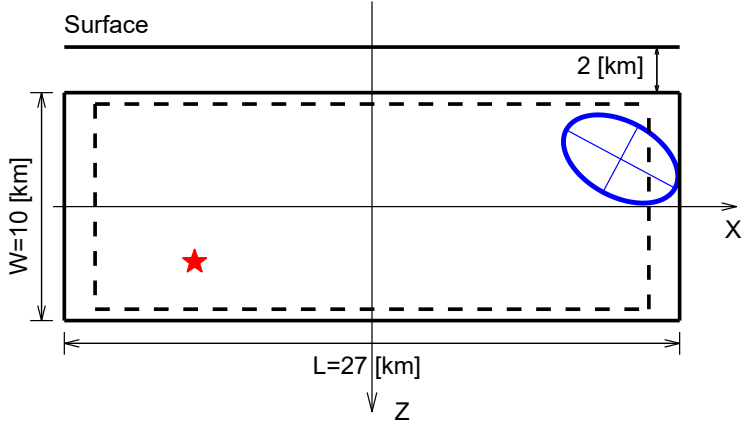

Figure 11. Inferred fault plane configuration with MCMC chain starting from the "symmetric" counterpart configuration.

differences are directly stemming from restrictions $\mathrm{R}-2$ and R-3.

Though it is not obvious to see from Fig. 13, the restricted Bayesian MCMC process is indeed aware of the existence of the "symmetric" counterpart configuration. Figure 14 shows the restricted Bayesian MCMC sample chains of both the hypocenter (Fig. 14a) and elliptical patch center (Fig. 14b). It is seen that despite the fact the hypocenter samples are mostly clustered around $x_{\mathrm{h}}=5 \mathrm{~km}$, there is a sample cloud on the opposite side $\left(x_{\mathrm{h}}=-5 \mathrm{~km}\right)$, corresponding to the "symmetric" counterpart configuration discussed before. The sample cloud of the elliptical center also shows bimodal distributions, with primary cloud on the left $\left(x_{\mathrm{c}}<0\right)$ and secondary "symmetric" counterpart on the right (around $x_{\mathrm{c}}=5 \mathrm{~km}$ ). The correspondence between $x_{\mathrm{h}}$ and $x_{\mathrm{c}}$ is shown in Fig. $14 \mathrm{c}$, from which it is seen that when $x_{\mathrm{h}}$ is positive, $x_{\mathrm{c}}$ is more likely to be negative, and vice versa, suggesting that hypocenter and ellipse center are on the opposite side of the fault plane, as previous inference results suggested. Note that in this restricted Bayesian MCMC sampling, the total number of samples remains $10^{6}$. The ability to observe the "symmetric" counterpart clouds is probably due to the fact that by introducing the auxiliary parameter $\zeta$, we dramatically shrunk the sampling space (it is only a small subspace of the original unrestricted parameter space). As mentioned before, introducing the auxiliary parameter $\zeta$ leads to significant efficiency improvement in the MCMC sampling process.

\subsection{Comparing PGVs}

We summarize the Bayesian analysis by comparing PCpredicted PGV responses to the three inferred fault plane configurations discussed above with the reference GMPE curve (see Fig. 15 and Table 3). We observe that all three configurations lead to a relatively close match between PC predictions and the reference GMPE curve. By comparing either the root mean square (rms) error or the relative rms error (see Table 3), we conclude that the red dots (corresponding to the unrestricted inference in Fig. 11) clearly show larger discrepancy from the GMPE curve, suggesting smaller likelihood compared to the other two, consistent with our Bayesian analysis. When comparing the blue and green dots (unrestricted inference in Fig. 10 versus restricted inference in Fig. 13), the former seems to be slightly better, which is expected because of the additional flexibility in fitting the GMPE curve. Nevertheless, it might be better to report the restricted inference results (configuration in Fig. 13), as it satisfies all the restrictions learned from previous studies while retaining plausible agreement with the reference GMPE curve.

\section{Conclusions}

An earthquake rupture model was adopted to explore the stochastic dependence of ground motion (in terms of PGVs) on random fault plane configurations. Thanks to the ability to generate two independent source model simulation ensembles with 8000 members each, we were able to build successful PC surrogate models to assess PGV responses over the virtual network of $N_{\mathrm{obs}}=56$ stations from one ensemble, and then to validate the quality of PC models on the other. Our statistical analysis showed that the two 8000-member LHS ensembles of source model simulations are adequate to represent the underlying PGV distributions at all stations, as they closely match with PC-predicted distributions over a much larger sample set.

A global sensitivity analysis of PC surrogate models was conducted. The analysis revealed that the source model PGV response is primarily sensitive to the hypocenter location, and much less sensitive to properties of the asperity patch, especially at stations far away from the fault plane (in terms of the $R_{\mathrm{JB}}$ distance). While this holds true for all stations, it is noted that asperity patch properties still carry considerable impact (20-30\% associated variability) on PGV responses at stations close to the fault plane, and even more influence (additional $10 \%$ variability) if one takes into consideration the interaction between asperity patch and hypocenter location.

Our analysis of PGV variabilities indicated that one needs to be cautious when interpreting PGVs at near-fault-plane stations, as they are more prone to higher model noise. This is supported by the Bayesian inference analysis, in which four independent model noise parameters $\left(\sigma_{l}^{2}\right.$ for $\left.l=1,2,3,4\right)$ were introduced and assigned to four groups of observational stations, depending on their $R_{\mathrm{JB}}$ distances away from the fault plane. The Bayesian inference results clearly showed the decreasing trend of noise parameters ( $\sigma_{l}^{2}$ values) when moving away from the fault plane (see Fig. 9). Further refinement of the noise parameter profile along the $R_{\mathrm{JB}}$ distance, though desired, is prohibited by the limited number of available observational stations.

We conducted both unrestricted and restricted Bayesian inference analyses to identify the chosen GMPE reference curve. The key findings are as follows. (1) Given the station distribution (Fig. 2) in this study, it is more likely to 


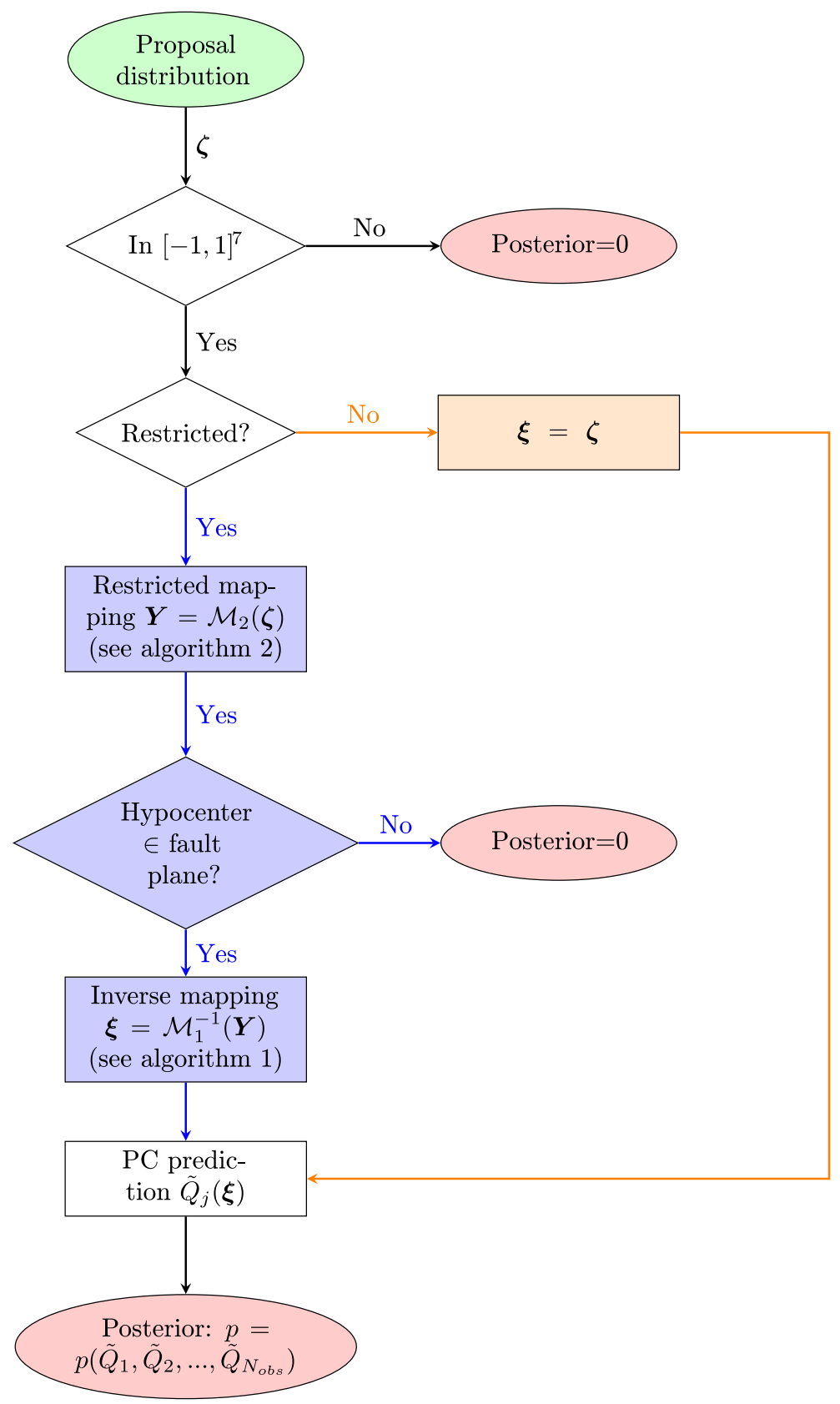

Figure 12. Flow chart demonstrating the random sampling process and the calculation of posterior probability in MCMC. The orange path corresponds to unrestricted sampling process, whereas the blue path incorporates additional restrictions on fault plane configurations. Note that $\boldsymbol{Y}$ denotes the fault plane configuration vector in the physical domain, e.g., $\boldsymbol{Y}=\left(\mathrm{AR}, x_{\mathrm{h}}, z_{\mathrm{h}}, a, \theta, x_{\mathrm{c}}, z_{\mathrm{c}}\right)^{T}$.

have the hypocenter located in the lower right quadrant of the fault plane, and the elliptical patch centered in the lower left quadrant. (2) Due to the considerable "symmetry" presented by those $N_{\text {obs }}$ stations, the most profound fault plane configuration, which best reproduce the reference GMPE predictions, can potentially have a "symmetric" twin configuration, especially for the hypocenter location. (3) The restricted inference results remain consistent with the unrestricted ones, with slightly more deviation from the chosen GMPE refer- ence curve. (4) Most importantly, our analyses suggest that the hypocenter and slip patch cannot be in the near-surface area of the fault, and they need to be some distance away from each other in order to produce the proper seismic radiation pattern, including on-fault directivity. Otherwise, the resulting near-source waveforms, and hence PGVs, would not match with GMPE results. This is consistent with the findings of Mai et al. (2005). 

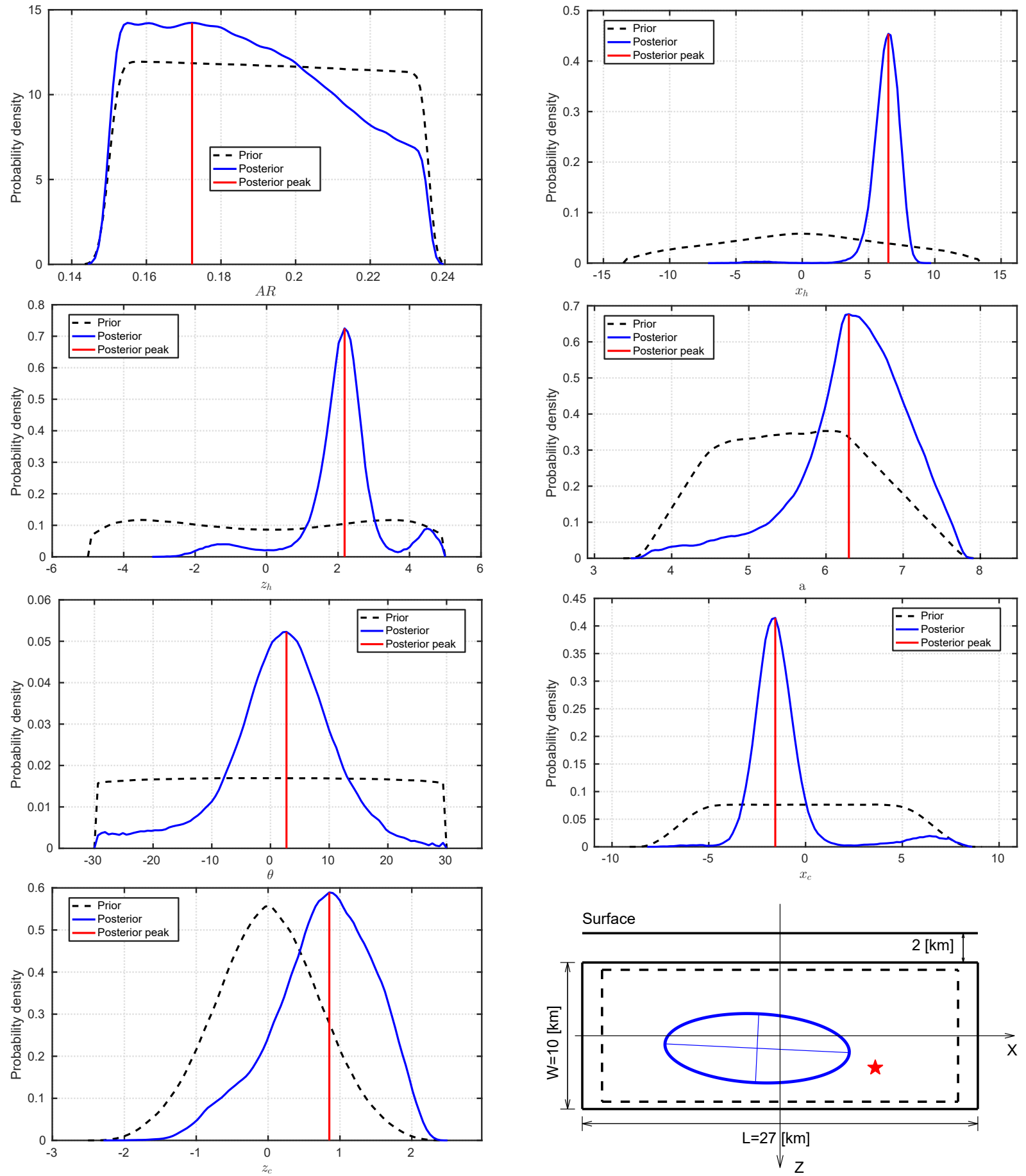

Figure 13. Prior (dashed black, derived from uniform $\zeta$ distribution in $\boldsymbol{\Xi}$ ) and posterior (solid blue) distributions of physical fault plane configuration parameters in restricted inference. The bottom right panel shows the inferred fault plane configuration.

The analyses and findings in this study provide useful insights on how near-source ground shaking (and its variability) depends on random fault rupture configurations. Interestingly, even very simple source models (with elliptical slip patches) are able to generate shaking distributions that well reproduce empirical predictions. To better reproduce the cho- sen GMPE reference curve, it might be beneficial to consider two or more asperity patches, instead of one in this study, in order to reduce the hypocenter location influence and in return increase the impact of asperity properties. Another potential improvement can be made by refining the station network. As mentioned earlier, the Bayesian inference is pri- 
Table 3. Comparison of PC-predicted PGVs of different inferred configurations with the reference GMPE curve. Unrestricted-1 and -2 correspond to inferences in Figs. 10 and 11, respectively. Each inferred configuration leads to PGV predictions in Fig. 15, as indicated by different colors.

\begin{tabular}{lcc}
\hline Inference & $\epsilon=\sqrt{\frac{\sum_{j=1}^{N_{\mathrm{obs}}\left(\widetilde{\mathcal{Q}}_{j}-\mathcal{Q}_{j}^{\mathrm{GMPE}}\right)^{2}}}{N_{\mathrm{obs}}}} \quad r=\sqrt{\frac{1}{N_{\mathrm{obs}}} \sum_{j=1}^{N_{\mathrm{obs}}\left(\frac{\widetilde{\mathcal{Q}}_{j}-\mathcal{Q}_{j}^{\mathrm{GMPE}}}{\mathcal{Q}_{j}^{\mathrm{GMPE}}}\right)^{2}}}$ \\
\hline Unrestricted-1 (blue) & 1.1135 & 0.3395 \\
Unrestricted-2 (red) & 1.7413 & 0.3993 \\
Restricted (green) & 1.4564 & 0.3702 \\
\hline
\end{tabular}
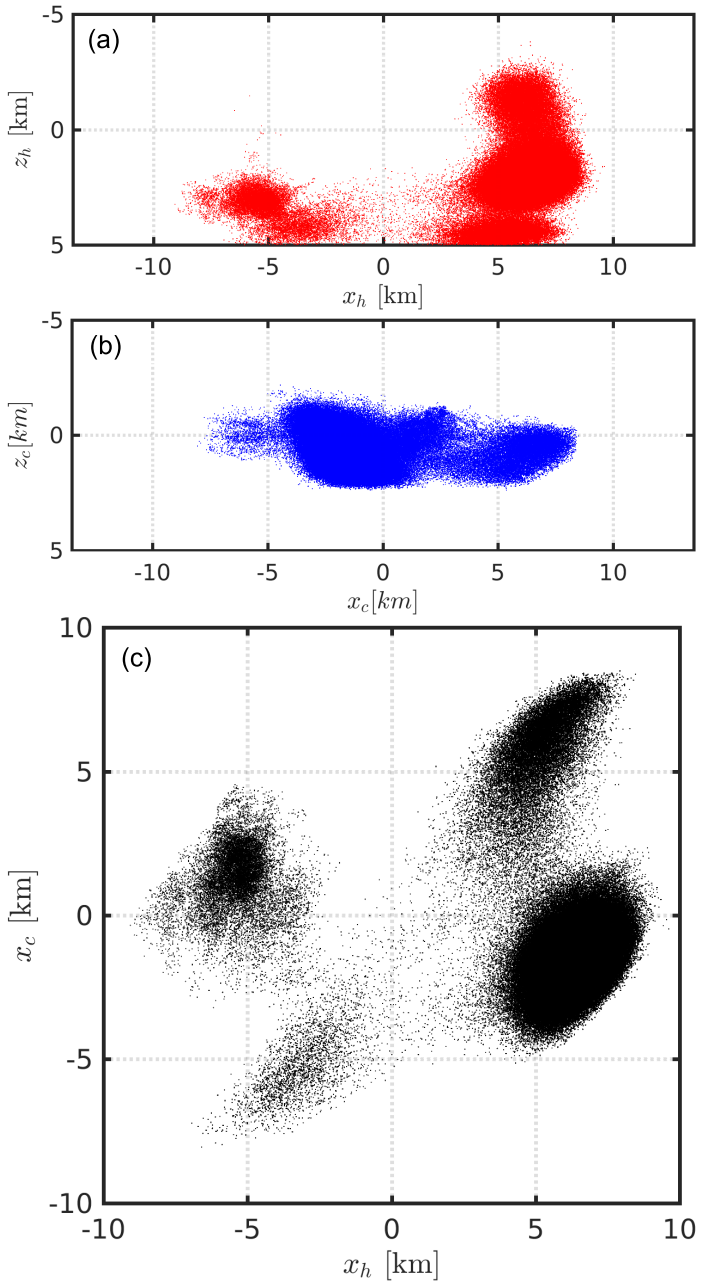

Figure 14. Restricted Bayesian MCMC sample chains of the hypocenter (a) and elliptical patch center (b); panel (c) shows the correspondence between $x_{\mathrm{h}}$ and $x_{\mathrm{c}}$ chains.

marily limited by the number of available stations at which PGVs are reported. By increasing the number of PGV reporting stations, one may improve the Bayesian inference results (e.g., removing the ambiguity in inferring the elliptical patch location).

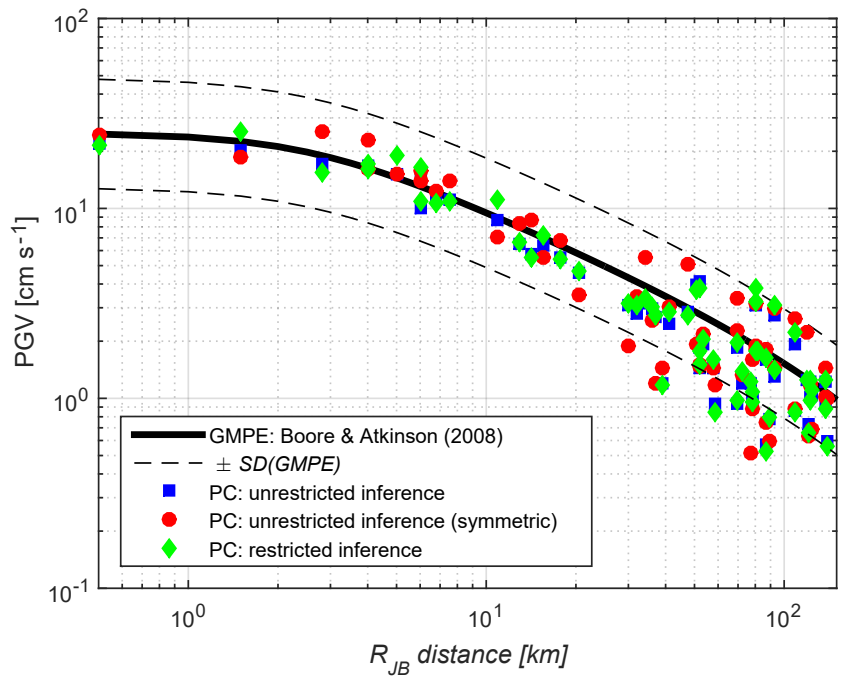

Figure 15. Comparison of PC-predicted PGV responses with aforementioned three inferred fault plane configurations with the reference GMPE curve. Dashed lines are standard deviation bounds of GMPE predictions.

Code and data availability. The COMPSYN code (Spudich and $\mathrm{Xu}, 2003)$ employed in this study has recently been made available at https://www.github.com/usgs; the rupture model simulation data in this study are available upon request. 
Appendix A: Mapping from PC random parameters to physical parameters

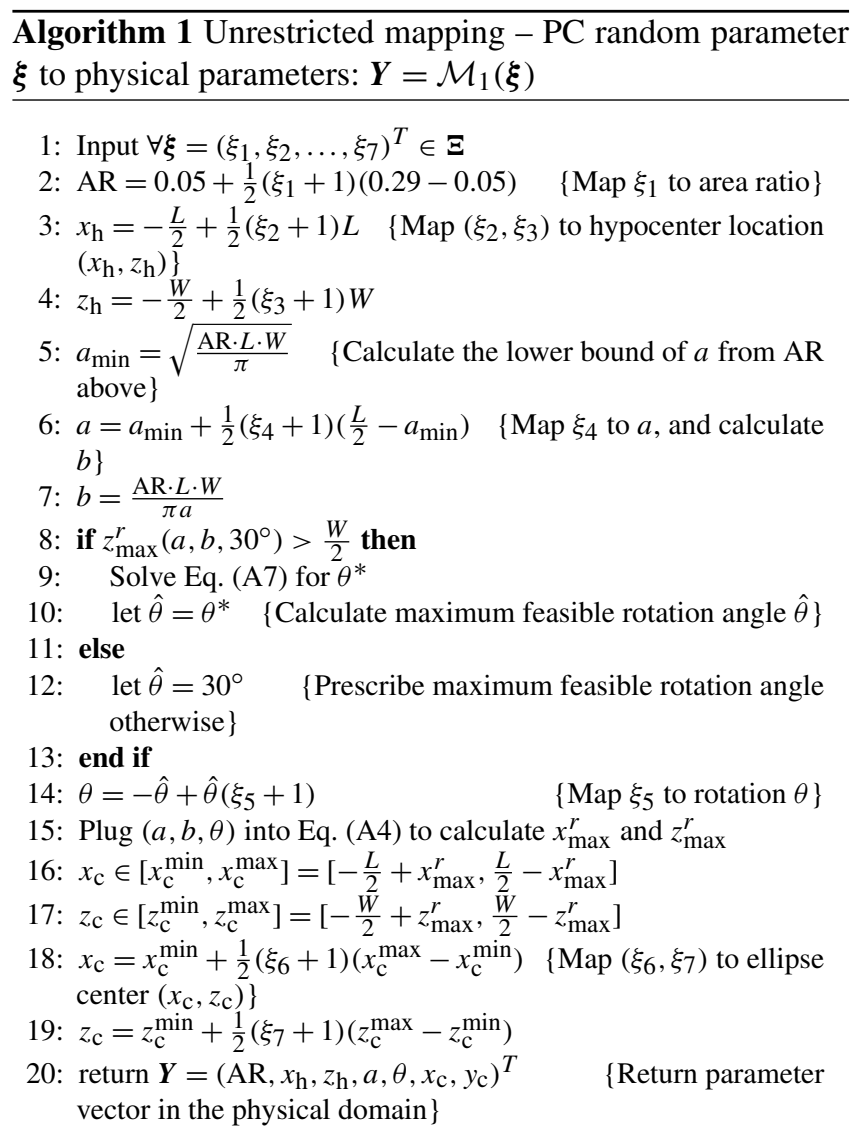

Let $a$ and $b$ be the lengths of semi-major and minor axes, respectively, of the elliptical patch considered in the fault plane configuration discussed in Sect. 2, and AR be the area ratio defined by $\mathrm{AR}=\frac{\pi a b}{L W}$ (here, $L=27 \mathrm{~km}$ and $W=10 \mathrm{~km}$ are the length and width of the fault plane). The elliptical patch centered at the origin $\left(x_{\mathrm{c}}=0\right.$ and $z_{\mathrm{c}}=0$, note the $z$ axis is pointing downwards as shown in Fig. 1), when not rotated (meaning $\theta=0$, the semi-major axis align with $x$ axis), can be expressed as

$\left[\begin{array}{l}x \\ z\end{array}\right]=\left[\begin{array}{l}a \cos \beta \\ b \sin \beta\end{array}\right]$ where $-\pi \leq \beta \leq \pi$.

If the elliptical patch is rotated by $\theta \in\left[-30^{\circ},+30^{\circ}\right]$ (a positive angle denotes clockwise rotation), then the ellipse is given by

$$
\begin{aligned}
{\left[\begin{array}{l}
x^{r} \\
z^{r}
\end{array}\right] } & =\left[\begin{array}{cc}
\cos \theta & -\sin \theta \\
\sin \theta & \cos \theta
\end{array}\right]\left[\begin{array}{l}
x \\
z
\end{array}\right] \\
& =\left[\begin{array}{cc}
\cos \theta & -\sin \theta \\
\sin \theta & \cos \theta
\end{array}\right]\left[\begin{array}{l}
a \cos \beta \\
b \sin \beta
\end{array}\right] \\
& =\left[\begin{array}{l}
a \cos \theta \cos \beta-b \sin \theta \sin \beta \\
a \sin \theta \cos \beta+b \cos \theta \sin \beta
\end{array}\right] .
\end{aligned}
$$

To ensure the resulting elliptical patch is completely confined within the fault plane, we first find the maximum extent of the ellipse in both $x$ and $y$ directions. We first calculate the following two $\beta^{*}$ values:

$$
\begin{aligned}
& \frac{\partial x^{r}}{\partial \beta}=-a \cos \theta \sin \beta-b \sin \theta \cos \beta=0 \\
& \Rightarrow \beta_{x}^{*}=\tan ^{-1}\left(-\frac{b}{a} \tan \theta\right) \\
& \frac{\partial z^{r}}{\partial \beta}=-a \sin \theta \sin \beta+b \cos \theta \cos \beta=0 \\
& \Rightarrow \beta_{z}^{*}=\tan ^{-1}\left(\frac{b}{a} \frac{1}{\tan \theta}\right) .
\end{aligned}
$$

Next, by substituting the above $\beta_{x}^{*}$ and $\beta_{z}^{*}$ into Eq. (A2), we have

$x_{\text {max }}^{r}=\left|a \cos \theta \cos \beta_{x}^{*}-b \sin \theta \sin \beta_{x}^{*}\right|$

$z_{\max }^{r}=\left|a \sin \theta \cos \beta_{z}^{*}+b \cos \theta \sin \beta_{z}^{*}\right|$.

These are the maximum extents of the ellipse in $x$ and $y$ directions, respectively.

When the ellipse is not centered at the origin $\left(x_{\mathrm{c}} \neq 0\right.$ and/or $z_{\mathrm{c}} \neq 0$ ), the following conditions need to be satisfied:

$\left|x_{\mathrm{c}}\right|+x_{\max }^{r} \leq \frac{L}{2}$

$\left|z_{\mathrm{c}}\right|+z_{\max }^{r} \leq \frac{W}{2}$,

which leads to

$$
\begin{aligned}
& \left|x_{\mathrm{c}}\right| \in\left[0, \frac{L}{2}-x_{\text {max }}^{r}\right] \\
& \left|z_{\mathrm{c}}\right| \in\left[0, \frac{W}{2}-z_{\max }^{r}\right] .
\end{aligned}
$$

Note that the above constraint on $x_{\mathrm{c}}$ is always valid, since $x_{\max }^{r} \leq a \leq \frac{L}{2}$, while the $z_{\mathrm{c}}$ constraint requires more treatment as $z_{\max }^{r}$ can be greater than $\frac{W}{2}$ under some rotation angle $\theta$ and semi-major axis $a$. To ensure that $z_{\max }^{r} \leq \frac{W}{2}$, we first check if the prescribed upper bound rotation $\left(30^{\circ}\right)$ is feasible. If not, we solve the following equation for $\theta^{*}$, which corresponds to the maximum feasible rotation angle given $a$ and AR:

$$
\begin{aligned}
z_{\max }^{r}= & \mid a \sin \theta^{*} \cos \beta_{z}^{*}\left(\theta^{*}, a, \mathrm{AR}\right) \\
& +b \cos \theta^{*} \sin \beta_{z}^{*}\left(\theta^{*}, a, \mathrm{AR}\right) \mid=\frac{W}{2},
\end{aligned}
$$

and define the upper bound of the rotation angle as

$\hat{\theta}=\min \left(\theta^{*}(\mathrm{PE}, a), 30^{\circ}\right)$. 
The resulting rotation angle parameter $\theta$ is then assumed to be uniformly distributed over $[-\hat{\theta}, \hat{\theta}]$.

The mapping from $\xi$ to physical parameters is outlined in the Algorithm 1. With the prior assumption of uniform distribution of $\boldsymbol{\xi}$ in $\boldsymbol{\Xi}$, the corresponding prior distributions of each physical parameter are shown in Fig. 10 (dashed black curves).

\section{Appendix B: Restricted mapping}

We introduce the auxiliary parameter vector $\zeta \in \boldsymbol{\Xi}$ and design the following mapping process to generate fault plane configuration samples that satisfy our prior configuration restrictions. For clarity, we list again the four restrictions below:

R-1. The elliptical patch is inside the dashed rectangle $\left(\left[L^{\prime}, W^{\prime}\right]=0.9 \times[L, W]\right)$ shown in Fig. 1.

R-2. The area of the elliptical patch (AR) is between 15 and $29 \%$ of the fault plane area, i.e., $0.15<\mathrm{AR}<0.29$.

R-3. The elliptical patch is not too elongated, i.e., $\frac{a}{b}<3$.

R-4. The hypocenter is located outside but near the elliptical patch, i.e., $x_{\mathrm{h}}=\left(a+3 \zeta_{\mathrm{h}_{1}}\right) \cos \left(2 \pi \zeta_{\mathrm{h}_{2}}\right)$ and $z_{\mathrm{h}}=$ $\left(b+b \frac{3}{a} \zeta_{\mathrm{h}_{1}}\right) \sin \left(2 \pi \zeta_{\mathrm{h}_{2}}\right) \forall\left(\zeta_{\mathrm{h}_{1}}, \zeta_{\mathrm{h}_{2}}\right) \in[0,1]^{2}$.

The mapping process is similar to the one in Algorithm 1, with necessary modifications to satisfy the above conditions. We outline the constrained mapping in Algorithm 2. Note that there is one additional condition needs to be verified, i.e., whether or not the hypocenter is inside the fault plane, as it is not guaranteed by the mapping process (this is also indicated in Fig. 12).

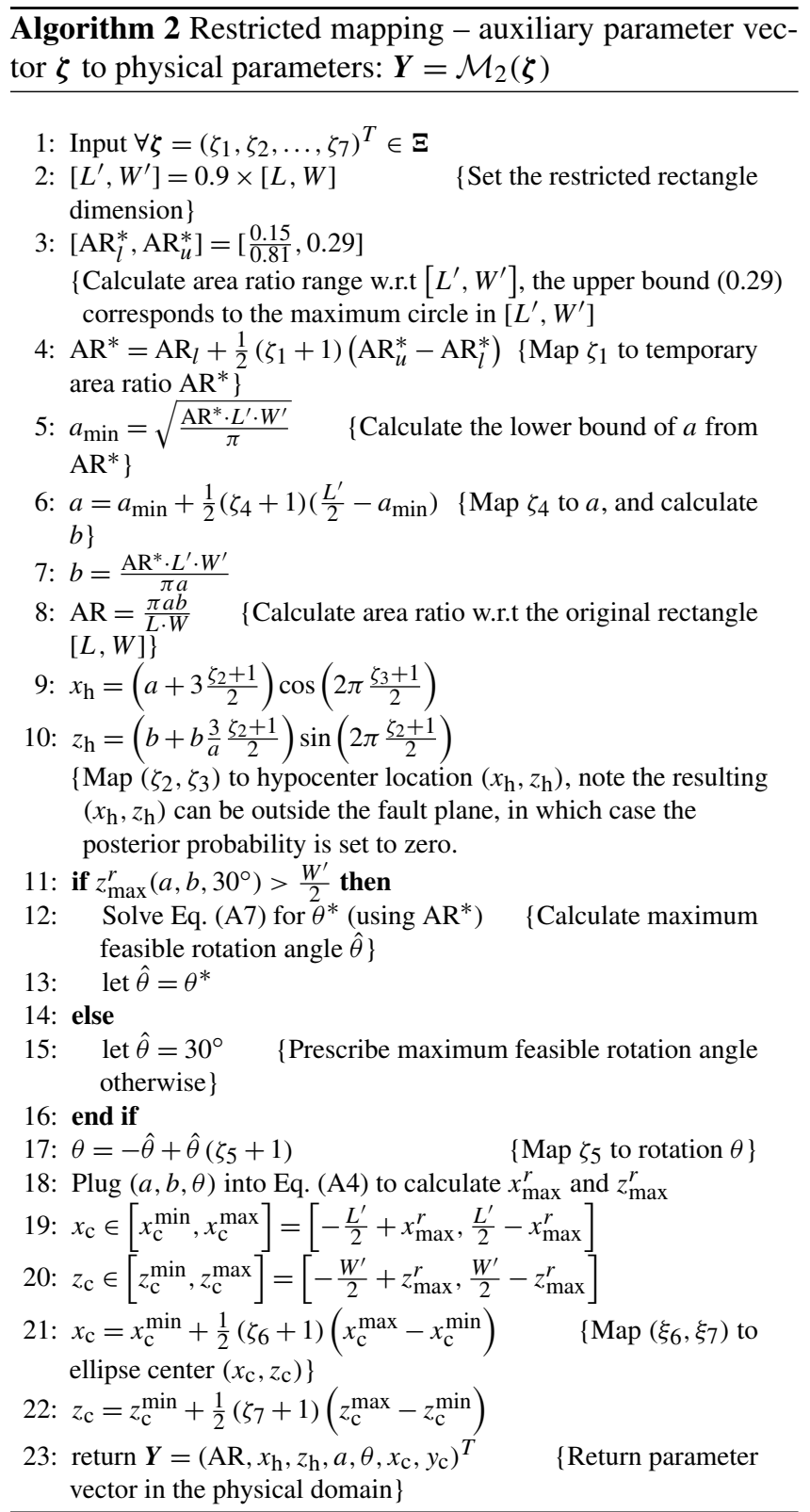


Author contributions. In this study, HCJ and PMM created the earthquake rupture model and generated both the training and validation ensembles of model simulations for building PC surrogates. The PC-based statistical analysis and Bayesian inference were conducted by GL and OMK. IH provided invaluable insights and advice throughout this work.

Competing interests. The authors declare that they have no conflict of interest.

Acknowledgements. Research reported in this publication was supported in part by research funding from King Abdullah University of Science and Technology (KAUST). The first author thanks KAUST for all the support during his postdoctoral fellowship. Earthquake rupture and ground-motion simulations have been carried out using the KAUST Supercomputing Laboratory (KSL) and we acknowledge the support of the KSL staff.

Edited by: Thomas Poulet

Reviewed by: two anonymous referees

\section{References}

Abrahamson, N. A., Silva, W. J., and Kamai, R.: Summary of the ASK14 ground motion relation for active crustal regions, Earthq. Spectra, 30, 1025-1055, 2014.

Alexanderian, A., Winokur, J., Sraj, I., Srinivasan, A., Iskandarani, M., Thacker, W. C., and Knio, O. M.: Global sensitivity analysis in an ocean general circulation model: a sparse spectral projection approach, Comput. Geosci., 16, 757-778, 2012.

Arroyo, D. and Ordaz, M.: Multivariate Bayesian regression analysis applied to ground-motion prediction equations, part 1: theory and synthetic example, B. Seismol. Soc. Am., 100, 1551-1567, 2010a.

Arroyo, D. and Ordaz, M.: Multivariate Bayesian regression analysis applied to ground-motion prediction equations, Part 2: Numerical example with actual data, B. Seismol. Soc. Am., 100, 1568-1577, 2010b.

Atkinson, G. M. and Boore, D. M.: Modifications to existing ground-motion prediction equations in light of new data, B. Seismol. Soc. Am., 101, 1121-1135, 2011.

Atkinson, G. M. and Silva, W.: Stochastic modeling of California ground motions, B. Seismol. Soc. Am., 90, 255-274, 2000.

Berger, J. O.: Statistical decision theory and Bayesian analysis, Springer Science \& Business Media, 2013.

Bernardo, J. M. and Smith, A. F. M.: Bayesian Theory, Meas. Sci. Technol., 12, 221, https://doi.org/10.1088/0957-0233/12/2/702, 2001.

Boore, D. M. and Atkinson, G. M.: Ground-Motion Prediction Equations for the Average Horizontal Component of PGA, PGV, and 5\%-Damped PSA at Spectral Periods between $0.01 \mathrm{~s}$ and $10.0 \mathrm{~s}$, Earthq. Spectra, 24, 99-138, https://doi.org/10.1193/1.2830434, 2008.

Boore, D. M., Joyner, W. B., and Fumal, T. E.: Equations for estimating horizontal response spectra and peak acceleration from western North American earthquakes: a summary of recent work, Seismol. Res. Lett., 68, 128-153, 1997.

Chiou, B., Darragh, R., Gregor, N., and Silva, W.: NGA project strong-motion database, Earthq. Spectra, 24, 23-44, 2008.

Cruz-Jiménez, H., Chávez-García, F. J., and Furumura, T.: Differences in attenuation of ground motion perpendicular to the mexican subduction zone between Colima and Guerrero: An explanation based on numerical modeling, B. Seismol. Soc. Am., 99, 400-406, 2009.

Debusschere, B., Sargsyan, K., Safta, C., and Chowdhary, K.: Uncertainty quantification toolkit (UQTk), Handbook of Uncertainty Quantification, 1-21, 2016.

Debusschere, B. J., Najm, H. N., Pébay, P. P., Knio, O. M., Ghanem, R. G., and Le Ma1tre, O. P.: Numerical challenges in the use of polynomial chaos representations for stochastic processes, SIAM J. Sci. Comput., 26, 698-719, 2004.

Furumura, T. and Singh, S.: Regional wave propagation from Mexican subduction zone earthquakes: The attenuation functions for interplate and inslab events, B. Seismol. Soc. Am., 92, 2110 2125, 2002.

Gelman, A., Carlin, J. B., Stern, H. S., and Rubin, D. B.: Bayesian data analysis, vol. 2, Chapman \& Hall/CRC Boca Raton, FL, USA, 2014.

Ghanem, R. G. and Spanos, P. D.: Stochastic finite elements: a spectral approach, Springer-Verlag New York, 1991.

Giraldi, L., Le Maître, O. P., Mandli, K. T., Dawson, C. N., Hoteit, I., and Knio, O. M.: Bayesian inference of earthquake parameters from buoy data using a polynomial chaos-based surrogate, Comput. Geosci., 21, 683-699, 2017.

Haario, H., Saksman, E., and Tamminen, J.: An adaptive Metropolis algorithm, Bernoulli, 223-242, 2001.

Homma, T. and Saltelli, A.: Importance measures in global sensitivity analysis of nonlinear models, Reliab. Eng. Syst. Safe., 52, 1-17, 1996.

Irikura, K. and Miyake, H.: Recipe for predicting strong ground motion from crustal earthquake scenarios, Pure Appl. Geophys., 168, 85-104, 2011.

Le Maître, O. P. and Knio, O. M.: Spectral methods for uncertainty quantification: with applications to computational fluid dynamics, Springer Science \& Business Media, 2010.

Mahani, A. B. and Atkinson, G. M.: Evaluation of functional forms for the attenuation of small-to-moderate-earthquake response spectral amplitudes in North America, B. Seismol. Soc. Am., 102, 2714-2726, 2012

Mai, P. M.: Ground motion: Complexity and scaling in the near field of earthquake ruptures, in: Encyclopedia of Complexity and Systems Science, Springer, 4435-4474, 2009.

Mai, P. M. and Beroza, G. C.: Source scaling properties from finitefault-rupture models, B. Seismol. Soc. Am., 90, 604-615, 2000.

Mai, P. M., Spudich, P., and Boatwright, J.: Hypocenter locations in finite-source rupture models, B. Seismol. Soc. Am., 95, 965-980, 2005.

Maufroy, E., Chaljub, E., Hollender, F., Kristek, J., Moczo, P., Klin, P., Priolo, E., Iwaki, A., Iwata, T., Etienne, V., De Martin, F., Theodoulidis, N., Manakou, M., Guyonnet-Benaize, C., and Bard, P.-Y.: Earthquake ground motion in the Mygdonian basin, Greece: the E2VP verification and validation of 3D numerical simulation up to $4 \mathrm{~Hz}$, B. Seismol. Soc. Am., 105, 1398-1418, 2015. 
Maufroy, E., Chaljub, E., Hollender, F., Bard, P.-Y., Kristek, J., Moczo, P., De Martin, F., Theodoulidis, N., Manakou, M., Guyonnet-Benaize, C., Hollard, N., and Pitilakis, K.: 3D numerical simulation and ground motion prediction: Verification, validation and beyond-Lessons from the E2VP project, Soil Dyn. Earthq. Eng., 91, 53-71, 2016.

McKay, M. D., Beckman, R. J., and Conover, W. J.: Comparison of three methods for selecting values of input variables in the analysis of output from a computer code, Technometrics, 21, 239-245, 1979.

Minson, S., Simons, M., Beck, J., Ortega, F., Jiang, J., Owen, S., Moore, A., Inbal, A., and Sladen, A.: Bayesian inversion for finite fault earthquake source models-II: the 2011 great Tohokuoki, Japan earthquake, Geophys. J. Int., 198, 922-940, 2014.

Olson, A. H., Orcutt, J. A., and Frazier, G. A.: The discrete wavenumber/finite element method for synthetic seismograms, Geophy. J. Int., 77, 421-460, 1984.

Roberts, G. O. and Rosenthal, J. S.: Examples of adaptive MCMC, J. Comput. Graph. Stat., 18, 349-367, 2009.

Seber, G. A. and Lee, A. J.: Linear regression analysis, vol. 329, John Wiley \& Sons, 2012.

Sheather, S. J. and Jones, M. C.: A reliable data-based bandwidth selection method for kernel density estimation, J. Roy. Stat. Soc. B Met., 53, 683-690, 1991.

Singh, S., Srinagesh, D., Srinivas, D., Arroyo, D., Pérez-Campos, X., Chadha, R., and Suresh, G.: Strong Ground Motion in the Indo-Gangetic Plains during the 2015 Gorkha, Nepal, Earthquake Sequence and Its Prediction during Future Earthquakes, B. Seismol. Soc. Am., 107, 1293-1306, 2017.

Sivia, D. and Skilling, J.: Data analysis: a Bayesian tutorial, OUP Oxford, 2006.

Sobol, I.: Sensitivity estimates for nonlinear mathematical models, Math. Model. Comput. Exp., 1, 407-414, 1993.

Somerville, P. G., Smith, N. F., Graves, R. W., and Abrahamson, N. A.: Modification of empirical strong ground motion attenuation relations to include the amplitude and duration effects of rupture directivity, Seismol. Res. Lett., 68, 199-222, 1997.
Somerville, P., Irikura, K., Graves, R., Sawada, S., Wald, D., Abrahamson, N., Iwasaki, Y., Kagawa, T., Smith, N., and Kowada, A.: Characterizing crustal earthquake slip models for the prediction of strong ground motion, Seismol. Res. Lett., 70, 59-80, 1999.

Spudich, P. and Xu, L.: 85.14-Software for Calculating Earthquake Ground Motions from Finite Faults in Vertically Varying Media, Int. Geophys., 81, 1633-1634, 2003.

Sraj, I., Mandli, K. T., Knio, O. M., Dawson, C. N., and Hoteit, I.: Quantifying Uncertainties in Fault Slip Distribution during the Tōhoku Tsunami using Polynomial Chaos, Ocean Dynam., 67, 1535-1551, https://doi.org/10.1007/s10236-017-1105-9, 2016.

Sudret, B. and Mai, C.: Computing seismic fragility curves using polynomial chaos expansions, in: Proc. 11th Int. Conf. Struct. Safety and Reliability (ICOSSAR-2013), Elsevier, New York, USA, 16-20 June 2013.

Thingbaijam, K. K. S., Martin Mai, P., and Goda, K.: New Empirical Earthquake Source-Scaling Laws, B. Seismol. Soc. Am., 107 2225-2246, 2017.

Tinti, E., Fukuyama, E., Piatanesi, A., and Cocco, M.: A kinematic source-time function compatible with earthquake dynamics, B. Seismol. Soc. Am., 95, 1211-1223, 2005.

Van Den Berg, E. and Friedlander, M.: SPGL1: A solver for largescale sparse reconstruction, available at: https://www.cs.ubc.ca/ $\sim$ mpf/spgl1/ (last access: 20 December 2017), 2007.

Van Den Berg, E. and Friedlander, M. P.: Probing the Pareto frontier for basis pursuit solutions, SIAM J. Sci. Comput., 31, 890-912, 2008.

Vyas, J. C., Mai, P. M., and Galis, M.: Distance and azimuthal dependence of ground-motion variability for unilateral strike-slip ruptures, B. Seismol. Soc. Am., 106, 1584-1599, 2016.

Wells, D. L. and Coppersmith, K. J.: New empirical relationships among magnitude, rupture length, rupture width, rupture area, and surface displacement, B. Seismol. Soc. Am., 84, 974-1002, 1994.

Xiu, D. and Karniadakis, G. E.: The Wiener-Askey polynomial chaos for stochastic differential equations, SIAM J. Sci. Comput., 24, 619-644, 2002. 